\title{
New hybrid scaffolds based on ASA/genistein: Synthesis, cytotoxic effect, molecular docking, drug-likeness, and in silico ADME/Tox modeling
}

\author{
Lizeth Gómez-R, Gustavo Moreno-Q, Angie Herrera-R*, Wilson Castrillón-L, Andrés F. Yepes, Wilson Cardona-G* \\ Química de Plantas Colombianas, Institute of Chemistry, Faculty of Exact and Natural Sciences, University of Antioquia, Medellín, Colombia.
}

\begin{tabular}{l}
\hline ARTICLE INFO \\
\hline Received on: $16 / 06 / 2021$ \\
Accepted on: 26/08/2021 \\
Available Online: 05/02/2022 \\
\hline Key words: \\
Genistein, acetylsalicylic acid \\
(ASA), hybrids, cytotoxicity, \\
colorectal cancer, in silico \\
ADME/Tox profile, molecular \\
docking.
\end{tabular}

\begin{abstract}
Eight hybrids based on genistein and aspirin were designed, synthesized, and elucidated by spectroscopic analysis. Chemopreventive potential of these compounds was evaluated against SW480 human colon adenocarcinoma cells, its metastatic derivative SW620 and normal cells (CHO-K1). According to the results, hybrids 4a and 4c displayed significant activity over SW480 cells causing a reduction of cell viability of $40.49 \%$ and $40.39 \%$, respectively, when treated with $300 \mu \mathrm{M}$ for 48 hours, being time- and concentration-dependent. None of the compounds exhibited activity at 24 hours; besides, these types of molecules were not active on human metastatic SW620 cells (data not shown). On the other hand, compounds $\mathbf{4 d}, \mathbf{4 e}, \mathbf{4 f}$, and $\mathbf{4 h}$ did not display any activity on the cells at the conditions evaluated. Multitarget docking studies performed against six tumor-associated proteins targets in colorectal cancer, would reveal that hybrids 4a and $\mathbf{4} \mathbf{c}$ may inhibit function of Polo-like kinase 1 as primary mode of action with a binding affinity about $-10 \mathrm{kcal} /$ mol. However, docking action would suggest these compounds may induce synergistic cytotoxic effects by regulation of B-cell lymphoma 2 (Bcl-2)/Bcl-2-associated X protein system and cyclooxygenase-2 in SW480 colorectal cancer cells with Vina scores in the range -10.1 to $-9.3 \mathrm{kcal} / \mathrm{mol}$. Additionally, in silico drug-likeness studies suggest that active hybrids $\mathbf{4 a}$ and $\mathbf{4 c}$ display favorable pharmacokinetics indices, good Absorption, Distribution, Metabolism and Excretion (ADME)/Tox profile, and a remarkable metabolic stability within recommended therapeutic ranges, making these compounds useful as promising medicinal scaffolds in the designing of novel orally active anticancer candidates.
\end{abstract}

\section{INTRODUCTION}

Colorectal cancer (CRC) continues to be a major cause of morbidity and mortality worldwide, being the third most incident and the second most mortal cancer in both sexes (Global Cancer Statistics, 2020). Current treatments for this disease incorporate combination chemotherapy mainly composed of 5-fluorouracil. Examples of these include FOLFIRI (5-FU/folinic acid/irinotecan), FOLFOXIRI (leucovorin/5-FU/oxaliplatin/irinotecan), and

\footnotetext{
*Corresponding Author

Angie Herrera-R, Química de Plantas Colombianas, Institute of Chemistry, Faculty of Exact and Natural Sciences, University of Antioquia, Medellin, Colombia.E-mail: angie.herrerar@udea.edu.co

Wilson Cardona-G, Quimica de Plantas Colombianas, Institute of Chemistry, Faculty of Exact and Natural Sciences, University of Antioquia, Medellin, Colombia.E-mail: wilson.cardona1@udea.edu.co
}

FOLFOX (5-FU/leucovorin/oxaliplatin). The use of these drugs is effective; however, they produce undesirable harmful effects associated with gastrointestinal and neurological disorders which many times cause limitations in the dose or discontinuation of anticancer therapy (McQuade et al., 2014; Pointet et al., 2017; Rejhova et al., 2018). Due to the increase in the statistics and the toxicity associated with conventional chemotherapy, extensive research is ongoing to develop new pharmaceutical agents with chemopreventive potential against colorectal cancer.

Genistein (Fig. 1) is a major metabolite of soy (a plant belonging to the Leguminosae family), which has displayed a wide range of biological activities such as antioxidant (Rahman Mazumder and Hongsprabhas, 2016), antiviral (LeCher et al., 2019), antiallergic (Mastuda et al., 2002), anti-inflammatory (Spagnuolo et al., 2018), antiestrogenic (Wang et al., 2006), and antibacterial (Satish et al., 2018). Furthermore, this compound has also exhibited anti-cancer activity (Ganai et al., 2015; Yang et al., 
2016). Chemopreventive potential attributed to genistein and its related flavonoids is due to the capability to modulate signaling pathways and several epigenetic markers that contribute to develop human colorectal carcinogenesis. In fact, epidemiological studies have shown that genistein displays activity against colorectal cancer initiation and progression, in different cell lines (HCT-116, SW-480, and HT-29) (Chae et al., 2019; Tuli et al., 2019), through activation of apoptotic executioner molecules, such as the nuclear factor-kappa B (NF-kB), tumor necrosis factor (TNF- $\alpha$ ) (Luo et al., 2014), B-cell lymphoma 2 (Bcl-2), Bcl-2-associated X protein (Bax), as well as by modulating the protease ability of caspase- 3 (Zhou et al., 2017), inducing mitotic arrest for inhibiting Pololike kinase 1 (Plk1) activity (Shin et al., 2017), and inhibiting the activity of cyclooxygenase-2 (COX-2) (Zhang et al., 2017).

On the other hand, aspirin, also known as acetylsalicylic acid (ASA) (Fig. 1), is a non-steroidal anti-inflammatory drug (NSAID), which has long been used as analgesic and antiinflammatory agent (Alqahtani et al., 2018). This compound inhibits the COX activity; besides, its chemopreventive potential has been reported, particularly in colon cancer (Jiang et al., 2020). Epidemiological studies that include both men and women revealed that aspirin reduced the incidence of CRC by about $70 \%$. This percentage increases with prolonged duration of aspirin intake (Avivi et al., 2012). Additionally, clinical studies have demonstrated a decrease in the mortality rate by up to $48 \%$ after continuous aspirin intake in diagnosed CRC patients (Avivi et al., 2012).

Nowadays, an emerging strategy for new drug discovery relies in the use of hybrid molecules which results from the covalent linking of two molecules with individual intrinsic pharmacological activity (Cardona et al., 2021). Hybrid molecules bear two distinct pharmacophores with different biological functions and may display dual activity, although both entities of the hybrid molecule are not necessarily acting on the same biological target (Cardona et al., 2018; de Oliveira Pedrosa et al., 20017).

In search for new alternatives to treat colorectal cancer, authors designed and synthesized a series of ASA-Genistein hybrids (Fig. 1), which were obtained via click reaction between different ASA-alkylazides and propargyl-genistein. In addition, it was conducted an oncoinformatics study to predict the binding affinity of promising hybrids through an exhaustive drug-likeness and in silico ADME/Tox modeling against six tumor-associated proteins targets in $\mathrm{CRC}$, to propose a plausible biochemical mechanism by which these compounds exert a significant inhibitory effect on SW480 cell growth.

\section{MATERIALS AND METHODS}

\section{Chemical synthesis}

General remarks

Genistein (synthetic, >95\%) was purchased from AK Scientific Chemicals (USA). Aspirin ( $\geq 99.0 \%$, Acetylsalicylic acid) was purchased from Sigma-Aldrich (Germany). An ultrasound equipment (BRANSON) was used to assist reactions. ${ }^{1} \mathrm{H}$ and ${ }^{13} \mathrm{C}$ NMR spectra were obtained on a Varian equipment. As reference was used, the signals of the deuterated solvents and the chemical shifts $(\delta)$ were displayed in ppm. TMS was used as an internal standard. Coupling constants $(J)$ are given in Hertz (Hz). HRMS was obtained using a Bruker Impact II UHR-Q-TOF mass spectrometry (Bruker Daltonik GmbH, Bremen Germany) in positive mode. For column chromatography and thin layer chromatography was used silica gel 60 (0.063-0.200 mesh, Merck, Whitehouse Station, NJ) and precoated silica gel plates (Merck 60 F254 $0.2 \mathrm{~mm}$ ).

\section{General procedure for the synthesis of ASA-bromoalkyl (1a-h).}

The titled compounds were synthesized following a procedure described in the literature (Lin et al., 2013): in a flatbottomed flask of $25 \mathrm{ml}$ equipped with a magnetic stirring bar, aspirin (1 mmol), N,N-Diisopropylethylamine (DIPEA) (1.5 $\mathrm{mmol}$ ), and N,N-Dimethylformamide (DMF) $(10 \mathrm{ml})$ were placed. The mixture was stirred for a period of 30 minutes. Then, 1, $\omega$-dibromoalkane $(1.2 \mathrm{mmol})$ was added to the reaction mixture and stirred for 3 hours. Water was added to reaction mixture and then it was extracted with ethyl acetate. Organic phase was dried on anhydrous sodium sulfate, filtered, concentrated under reduced pressure on a rotatory evaporator and the residue was purified by column chromatography over silica gel eluting with a mixture of hexane:ethyl acetate (ratio 8:2), to obtain bromoalkyl derivatives in yields ranging between $32 \%$ and $57 \%$.

2-bromoethyl 2-acetoxybenzoate (1a): yellow oil, 51\%. ${ }^{1} \mathrm{H}-\mathrm{NMR}$ (300 MHz, Chloroform-d) $\delta 8.11$ (dd, $J=7.8,1.8 \mathrm{~Hz}$, $1 \mathrm{H}), 7.63(\mathrm{ddd}, J=8.2,7.8,1.8 \mathrm{~Hz}, 1 \mathrm{H}), 7.38(\mathrm{td}, J=7.7,1.2 \mathrm{~Hz}$, $1 \mathrm{H}), 7.17(\mathrm{dd}, J=8.2,1.2 \mathrm{~Hz}, 1 \mathrm{H}), 4.62(\mathrm{t}, J=6.1 \mathrm{~Hz}, 2 \mathrm{H}), 3.65$ (t, $J=6.1 \mathrm{~Hz}, 2 \mathrm{H}), 2.41$ (s, 3H). ${ }^{13} \mathrm{C}-\mathrm{NMR}(75 \mathrm{MHz}$, Chloroform-d) $\delta 169.71(\mathrm{C}=\mathrm{O}), 163.86(\mathrm{C}=\mathrm{O}), 150.88,134.31,131.92,126.16$, $123.93,122.63,64.40,28.64,21.14$.
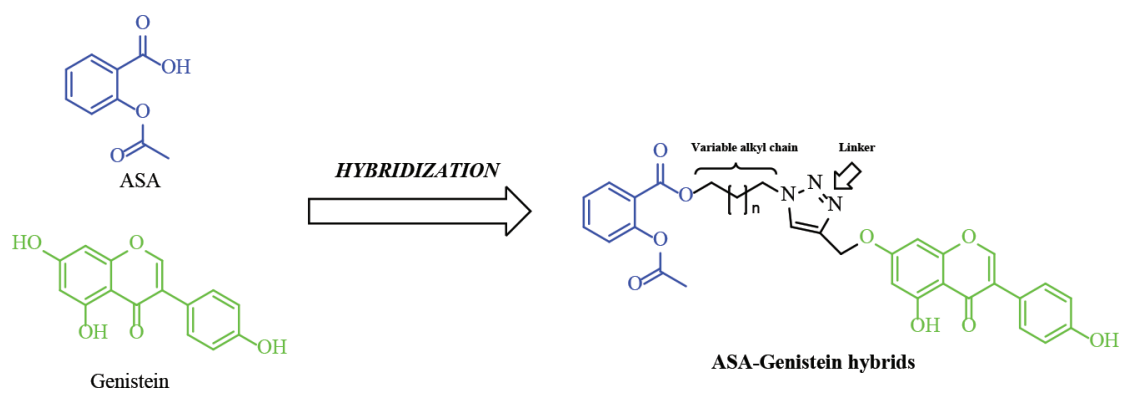

Figure 1. Design of ASA-Genistein hybrids. 
3-bromopropyl 2-acetoxybenzoate (1b): yellow oil, 57\%. ${ }^{1} \mathrm{H}-\mathrm{NMR}(300 \mathrm{MHz}$, Chloroform-d) $\delta 8.05$ (dd, $J=7.8,1.8 \mathrm{~Hz}$, $1 \mathrm{H}), 7.62(\mathrm{td}, J=8.1,1.8 \mathrm{~Hz}, 1 \mathrm{H}), 7.36(\mathrm{td}, J=7.7,1.2 \mathrm{~Hz}, 1 \mathrm{H})$, $7.16(\mathrm{dd}, J=8.1,1.2 \mathrm{~Hz}, 1 \mathrm{H}), 4.47(\mathrm{t}, J=6.0 \mathrm{~Hz}, 2 \mathrm{H}), 3.56(\mathrm{t}, J=$ $6.5 \mathrm{~Hz}, 2 \mathrm{H}), 2.43(\mathrm{~s}, 3 \mathrm{H}), 2.38-2.25(\mathrm{~m}, 2 \mathrm{H}) .{ }^{13} \mathrm{C}-\mathrm{NMR}(75 \mathrm{MHz}$, Chloroform-d) $\delta 169.75(\mathrm{C}=\mathrm{O}), 164.26(\mathrm{C}=\mathrm{O}), 150.74,134.09$, $131.71,126.11,123.91,123.06,62.83,31.75,29.42,21.13$.

4-bromobutyl 2-acetoxybenzoate (1c): yellow oil, 56\%. ${ }^{1} \mathrm{H}-\mathrm{NMR}(300 \mathrm{MHz}$, Chloroform-d) $\delta 8.05$ (dd, $J=7.8,1.8 \mathrm{~Hz}$, $1 \mathrm{H}), 7.61(\mathrm{td}, J=8.1,1.8 \mathrm{~Hz}, 1 \mathrm{H}), 7.36(\mathrm{td}, J=7.8,1.2 \mathrm{~Hz}, 1 \mathrm{H})$, $7.15(\mathrm{dd}, J=8.1,1.2 \mathrm{~Hz}, 1 \mathrm{H}), 4.35(\mathrm{t}, J=6.1 \mathrm{~Hz}, 2 \mathrm{H}), 3.51(\mathrm{t}, J=$ $6.3 \mathrm{~Hz}, 2 \mathrm{H}), 2.40(\mathrm{~s}, 3 \mathrm{H}), 2.13-1.87(\mathrm{~m}, 4 \mathrm{H}) .{ }^{13} \mathrm{C}-\mathrm{NMR}(75 \mathrm{MHz}$, Chloroform-d) $\delta 169.74(\mathrm{C}=\mathrm{O}), 164.40(\mathrm{C}=\mathrm{O}), 150.73,133.98$, $131.67,126.09,123.87,123.21,64.14,33.15,29.25,27.36,21.13$.

5-bromopentyl 2-acetoxybenzoate (1d): yellow oil, 53\%. ${ }^{1} \mathrm{H}-\mathrm{NMR}(300 \mathrm{MHz}$, Chloroform-d) $\delta 8.06$ (dd, $J=7.8,1.8 \mathrm{~Hz}$, $1 \mathrm{H}), 7.60(\mathrm{ddd}, J=8.1,7.4,1.8 \mathrm{~Hz}, 1 \mathrm{H}), 7.36(\mathrm{td}, J=7.6,1.3 \mathrm{~Hz}$, $1 \mathrm{H}), 7.15(\mathrm{dd}, J=8.1,1.3 \mathrm{~Hz}, 1 \mathrm{H}), 4.33$ (t, $J=6.6 \mathrm{~Hz}, 2 \mathrm{H}), 3.47$ $(\mathrm{t}, J=6.7 \mathrm{~Hz}, 2 \mathrm{H}), 2.40(\mathrm{~s}, 3 \mathrm{H}), 2.03-1.90(\mathrm{~m}, 2 \mathrm{H}), 1.88-1.76$ (m, 2H), 1.73-1.56 (m, 2H). ${ }^{13} \mathrm{C}-\mathrm{NMR}(75 \mathrm{MHz}$, Chloroform-d) $\delta 169.73(\mathrm{C}=\mathrm{O}), 164.46(\mathrm{C}=\mathrm{O}), 150.71,133.90,131.70,126.07$, $123.84,123.33,64.81,33.51,32.31,27.88,24.67,21.12$.

6-bromohexyl 2-acetoxybenzoate (1e): yellow oil, 55\%. ${ }^{1} \mathrm{H}-\mathrm{NMR}(300 \mathrm{MHz}$, Chloroform-d) $\delta 8.06$ (dd, $J=7.8,1.7 \mathrm{~Hz}$, $1 \mathrm{H}), 7.60(\mathrm{td}, J=8.1,1.7 \mathrm{~Hz}, 1 \mathrm{H}), 7.36(\mathrm{td}, J=7.7,1.1 \mathrm{~Hz}, 1 \mathrm{H})$, $7.15(\mathrm{dd}, J=8.1,1.1 \mathrm{~Hz}, 1 \mathrm{H}), 4.32(\mathrm{t}, J=6.7 \mathrm{~Hz}, 2 \mathrm{H}), 3.46(\mathrm{t}, J=$ $6.7 \mathrm{~Hz}, 2 \mathrm{H}), 2.40$ (s, 3H), 1.99-1.87 (m, 2H), 1.86-1.73 (m, 2H), 1.62-1.43 (m, 4H). ${ }^{13} \mathrm{C}-\mathrm{NMR}(75 \mathrm{MHz}$, Chloroform-d) $\delta 169.72$ $(\mathrm{C}=\mathrm{O}), 164.51(\mathrm{C}=\mathrm{O}), 150.69,133.86,131.72,126.06,123.84$, $123.40,65.03,33.79,32.63,28.54,27.85,25.23,21.12$.

8-bromooctyl 2-acetoxybenzoate (1f): yellow oil, 51\%. ${ }^{1} \mathrm{H}-\mathrm{NMR}(300 \mathrm{MHz}$, Chloroform-d) $\delta 8.06$ (dd, $J=7.8,1.7 \mathrm{~Hz}$, $1 \mathrm{H}), 7.60(\mathrm{td}, J=8.1,1.7 \mathrm{~Hz}, 1 \mathrm{H}), 7.36(\mathrm{td}, J=7.8,1.1 \mathrm{~Hz}, 1 \mathrm{H})$, $7.14(\mathrm{dd}, J=8.1,1.1 \mathrm{~Hz}, 1 \mathrm{H}), 4.31(\mathrm{t}, J=6.7 \mathrm{~Hz}, 2 \mathrm{H}), 3.45(\mathrm{t}, J=$ $6.8 \mathrm{~Hz}, 2 \mathrm{H}), 2.39$ (s, 3H), 1.96-1.84 (m, 2H), 1.83-1.68 (m, 2H), 1.54-1.33 (m, 8H). ${ }^{13} \mathrm{C}-\mathrm{NMR}$ (75 MHz, Chloroform-d) $\delta 169.73$ $(\mathrm{C}=\mathrm{O}), 164.55(\mathrm{C}=\mathrm{O}), 150.68,133.82,131.75,126.05,123.83$, $123.46,65.24,34.05,32.77,29.09,28.66,28.63,28.09,25.89$, 21.12 .

9-bromononyl 2-acetoxybenzoate (1g): yellow oil, $40 \%$. ${ }^{1} \mathrm{H}-\mathrm{NMR}(300 \mathrm{MHz}$, Chloroform-d) $\delta 8.06$ (dd, $J=7.8,1.6 \mathrm{~Hz}$, $1 \mathrm{H}), 7.60(\mathrm{td}, J=7.8,1.6 \mathrm{~Hz}, 1 \mathrm{H}), 7.36\left(\mathrm{t}_{\mathrm{app}}, J=7.6 \mathrm{~Hz}, 1 \mathrm{H}\right)$, $7.14\left(\mathrm{~d}_{\text {app }}, J=8.1 \mathrm{~Hz}, 1 \mathrm{H}\right), 4.31(\mathrm{t}, J=6.7 \mathrm{~Hz}, 2 \mathrm{H}), 3.45(\mathrm{t}, J=$ $6.8 \mathrm{~Hz}, 2 \mathrm{H}), 2.40(\mathrm{~s}, 3 \mathrm{H}), 1.95-1.83(\mathrm{~m}, 2 \mathrm{H}), 1.82-1.68(\mathrm{~m}, 2 \mathrm{H})$, $1.53-1.30(\mathrm{~m}, 10 \mathrm{H}) .{ }^{13} \mathrm{C}-\mathrm{NMR}(75 \mathrm{MHz}$, Chloroform-d) $\delta 169.73$ $(\mathrm{C}=\mathrm{O}), 164.56(\mathrm{C}=\mathrm{O}), 150.68,133.80,131.75,126.04,123.83$, $123.48,65.29,34.09,32.81,29.33,29.17,28.70,28.65,28.15$, $25.94,21.11$.

12-bromododecyl 2-acetoxybenzoate (1h): yellow oil, 32\%. ${ }^{1} \mathrm{H}-\mathrm{NMR}$ (300 MHz, Chloroform-d) $\delta 8.07$ (dd, $J=7.8,1.7$ $\mathrm{Hz}, 1 \mathrm{H}), 7.60(\mathrm{td}, J=8.1,1.7 \mathrm{~Hz}, 1 \mathrm{H}), 7.36(\mathrm{td}, J=7.7,1.1 \mathrm{~Hz}$, $1 \mathrm{H}), 7.14$ (dd, $J=8.1,1.1 \mathrm{~Hz}, 1 \mathrm{H}), 4.31$ (t, $J=6.8 \mathrm{~Hz}, 2 \mathrm{H}), 3.45$ $(\mathrm{t}, J=6.9 \mathrm{~Hz}, 2 \mathrm{H}), 2.40(\mathrm{~s}, 3 \mathrm{H}), 1.95-1.83(\mathrm{~m}, 2 \mathrm{H}), 1.82-1.68$ (m, 2H), 1.52-1.27 (m, 16H). ${ }^{13} \mathrm{C}-\mathrm{NMR}$ (75 MHz, Chloroform-d) $\delta 169.74(\mathrm{C}=\mathrm{O}), 164.58(\mathrm{C}=\mathrm{O}), 150.67,133.78,131.77,126.03$, $123.82,123.50,65.35,34.15,32.86,29.55,29.53,29.46,29.29$, $28.80,28.67,28.21,25.99,21.11$.
General procedure for the synthesis of ASA-alkylazides (2a-h).

Intermediates $\mathbf{2} \mathbf{a}-\mathbf{h}$ were synthesized following a procedure described in the literature (Ding et al., 2019): Compounds 1a-h (1 mmol), sodium azide ( $3 \mathrm{mmol})$, and DMF $(5 \mathrm{ml})$ were placed in a $10 \mathrm{ml}$ flat-bottomed flask. The mixture was sonicated for a period of 1 hour to $40^{\circ} \mathrm{C}$. Water was added to reaction mixture and then it was extracted with hexane. Organic phase was dried on anhydrous sodium sulfate, filtered, concentrated under reduced pressure on a rotatory evaporator to obtain pure azides $\mathbf{2 a}-\mathbf{h}$ in yields ranging between $72 \%$ and $96 \%$.

2-azidoethyl 2-acetoxybenzoate (2a): light yellow oil, 77\%, ${ }^{1} \mathrm{H}-\mathrm{NMR}$ (300 MHz, Chloroform-d) $\delta 8.09$ (dd, $J=7.8,1.8$ $\mathrm{Hz}, 1 \mathrm{H}), 7.63$ (ddd, $J=8.1,7.6,1.8 \mathrm{~Hz}, 1 \mathrm{H}), 7.37$ (td, $J=7.8$, $1.3 \mathrm{~Hz}, 1 \mathrm{H}), 7.16(\mathrm{dd}, J=8.1,1.3 \mathrm{~Hz}, 1 \mathrm{H}), 4.46(\mathrm{t}, J=5.2 \mathrm{~Hz}$, $2 \mathrm{H}), 3.62(\mathrm{t}, J=5.2 \mathrm{~Hz}, 2 \mathrm{H}), 2.41(\mathrm{~s}, 3 \mathrm{H}) .{ }^{13} \mathrm{C}-\mathrm{NMR}(75 \mathrm{MHz}$, Chloroform-d) $\delta 169.74(\mathrm{C}=\mathrm{O}), 163.99(\mathrm{C}=\mathrm{O}), 150.93,134.33$, 131.81, 126.18, 123.93, 122.55, 63.52, 49.86, 21.05.

3-azidopropyl 2-acetoxybenzoate (2b): light yellow oil, 74\%, ${ }^{1} \mathrm{H}-\mathrm{NMR}(300 \mathrm{MHz}$, Chloroform-d) $\delta 8.05$ (dd, $J=7.8,1.8$ $\mathrm{Hz}, 1 \mathrm{H}), 7.62(\mathrm{td}, J=8.1,1.8 \mathrm{~Hz}, 1 \mathrm{H}), 7.36(\mathrm{td}, J=7.8,1.2 \mathrm{~Hz}$, $1 \mathrm{H}), 7.16(\mathrm{dd}, J=8.1,1.2 \mathrm{~Hz}, 1 \mathrm{H}), 4.41(\mathrm{t}, J=6.2 \mathrm{~Hz}, 2 \mathrm{H}), 3.50$ $(\mathrm{t}, J=6.7 \mathrm{~Hz}, 2 \mathrm{H}), 2.39(\mathrm{~s}, 3 \mathrm{H}), 2.11-2.01(\mathrm{~m}, 2 \mathrm{H}) .{ }^{13} \mathrm{C}-\mathrm{NMR}$ (75 MHz, Chloroform-d) $\delta 169.74(\mathrm{C}=\mathrm{O}), 164.26(\mathrm{C}=\mathrm{O}), 150.75$, $134.08,131.64,126.10,123.89,123.07,61.95,48.14,28.25$, 21.08.

4-azidobutyl 2-acetoxybenzoate (2c): light yellow oil, $81 \%,{ }^{1} \mathrm{H}-\mathrm{NMR}(300 \mathrm{MHz}$, Chloroform-d) $\delta 8.05$ (dd, $J=7.9,1.8$ $\mathrm{Hz}, 1 \mathrm{H}), 7.61(\mathrm{td}, J=8,1,1.8 \mathrm{~Hz}, 1 \mathrm{H}), 7.36(\mathrm{td}, J=7.9,1.2 \mathrm{~Hz}$, $1 \mathrm{H}), 7.15(\mathrm{dd}, J=8.1,1.2 \mathrm{~Hz}, 1 \mathrm{H}), 4.35$ (t, $J=6.3 \mathrm{~Hz}, 2 \mathrm{H}), 3.40$ $(\mathrm{t}, J=6.5 \mathrm{~Hz}, 2 \mathrm{H}), 2.40(\mathrm{~s}, 3 \mathrm{H}), 1.95-1.83(\mathrm{~m}, 2 \mathrm{H}), 1.83-1.68(\mathrm{~m}$, 2H). ${ }^{13} \mathrm{C}-\mathrm{NMR}(75 \mathrm{MHz}$, Chloroform-d) $\delta 169.73(\mathrm{C}=\mathrm{O}), 164.39$ $(\mathrm{C}=\mathrm{O}), 150.73$, 133.97, 131.66, 126.08, 123.86, 123.21, 64.41, 51.03, 25.99, 25.61, 21.10.

5-azidopentyl 2-acetoxybenzoate (2d): light yellow oil, $87 \%,{ }^{1} \mathrm{H}-\mathrm{NMR}$ (300 MHz, Chloroform-d) $\delta 8.06(\mathrm{dd}, J=7.8,1.8$ $\mathrm{Hz}, 1 \mathrm{H}), 7.67-7.54$ (td, $J=8,1,1.8 \mathrm{~Hz}, 1 \mathrm{H}), 7.36$ (td, $J=7.8,1.2$ $\mathrm{Hz}, 1 \mathrm{H}), 7.15(\mathrm{dd}, J=8.1,1.2 \mathrm{~Hz}, 1 \mathrm{H}), 4.33(\mathrm{t}, J=6.6 \mathrm{~Hz}, 2 \mathrm{H})$, $3.34(\mathrm{t}, J=6.7 \mathrm{~Hz}, 2 \mathrm{H}), 2.39(\mathrm{~s}, 3 \mathrm{H}), 1.88-1.76(\mathrm{~m}, 2 \mathrm{H}), 1.76-1.64$ (m, 2H), 1.63-1.49 (m, 2H). ${ }^{13} \mathrm{C}-\mathrm{NMR}(75 \mathrm{MHz}$, Chloroform-d) $\delta 169.72(\mathrm{C}=\mathrm{O}), 164.46(\mathrm{C}=\mathrm{O}), 150.71,133.90,131.69,126.07$, 123.84, 123.33, 64.82, 51.27, 28.56, 28.27, 23.28, 21.10.

6-azidohexyl 2-acetoxybenzoate (2e): light yellow oil, $72 \%,{ }^{1} \mathrm{H}-\mathrm{NMR}(300 \mathrm{MHz}$, Chloroform-d) $\delta 8.06$ (dd, $J=7.9,1.8$ $\mathrm{Hz}, 1 \mathrm{H}), 7.60(\mathrm{td}, J=7.9,1.8 \mathrm{~Hz}, 1 \mathrm{H}), 7.36(\mathrm{td}, J=8.1,1.1 \mathrm{~Hz}$, $1 \mathrm{H}), 7.15$ (dd, $J=8.1,1.1 \mathrm{~Hz}, 1 \mathrm{H}), 4.32$ (t, $J=6.6 \mathrm{~Hz}, 2 \mathrm{H}), 3.32$ $(\mathrm{t}, J=6.8 \mathrm{~Hz}, 2 \mathrm{H}), 2.40(\mathrm{~s}, 3 \mathrm{H}), 1.86-1.74(\mathrm{~m}, 2 \mathrm{H}), 1.73-1.60$ (m, 2H), 1.56-1.41 (m, 4H). ${ }^{13} \mathrm{C}-\mathrm{NMR}$ (75 MHz, Chloroform-d) $\delta 169.73(\mathrm{C}=\mathrm{O}), 164.51(\mathrm{C}=\mathrm{O}), 150.69,133.86,131.71,126.06$, 123.84, 123.40, 65.02, 51.35, 28.78, 28.57, 26.44, 25.61, 21.10.

8-azidooctyl 2-acetoxybenzoate (2f): light yellow oil, 96\%, ${ }^{1} \mathrm{H}-\mathrm{NMR}(300 \mathrm{MHz}$, Chloroform-d) $\delta 8.06$ (dd, $J=7.8,1.8 \mathrm{~Hz}, 1 \mathrm{H})$, $7.60(\mathrm{td}, J=7.8,1.8 \mathrm{~Hz}, 1 \mathrm{H}), 7.36(\mathrm{td}, J=8.1,1.2 \mathrm{~Hz}, 1 \mathrm{H}), 7.15(\mathrm{dd}$, $J=8.1,1.2 \mathrm{~Hz}, 1 \mathrm{H}), 4.31(\mathrm{t}, J=6.7 \mathrm{~Hz}, 2 \mathrm{H}), 3.30(\mathrm{t}, J=6.9 \mathrm{~Hz}, 2 \mathrm{H})$, $2.40(\mathrm{~s}, 3 \mathrm{H}), 1.85-1.72(\mathrm{~m}, 2 \mathrm{H}), 1.71-1.57(\mathrm{~m}, 2 \mathrm{H}), 1.53-1.33(\mathrm{~m}$, $8 \mathrm{H}) .{ }^{13} \mathrm{C}-\mathrm{NMR}(75 \mathrm{MHz}$, Chloroform-d) $\delta 169.73(\mathrm{C}=\mathrm{O}), 164.55$ $(\mathrm{C}=\mathrm{O}), 150.68,133.81,131.74,126.04,123.83,123.47,65.24$, 51.46, 29.14, 29.06, 28.83, 28.63, 26.66, 25.89, 21.11 . 
9-azidononyl 2-acetoxybenzoate (2g): light yellow oil, 94\%, ${ }^{1} \mathrm{H}-\mathrm{NMR}(300 \mathrm{MHz}$, Chloroform-d) $\delta 8.06$ (dd, $J=7.8,1.8$ $\mathrm{Hz}, 1 \mathrm{H}), 7.60$ (ddd, $J=8.1,7.4,1.8 \mathrm{~Hz}, 1 \mathrm{H}), 7.35$ (td, $J=8.1,1.2$ $\mathrm{Hz}, 1 \mathrm{H}), 7.14(\mathrm{dd}, J=8.1,1.2 \mathrm{~Hz}, 1 \mathrm{H}), 4.31(\mathrm{t}, J=6.7 \mathrm{~Hz}, 2 \mathrm{H})$, $3.29(\mathrm{t}, J=6.9 \mathrm{~Hz}, 2 \mathrm{H}), 2.39(\mathrm{~s}, 3 \mathrm{H}), 1.85-1.69(\mathrm{~m}, 2 \mathrm{H}), 1.69-1.56$ (m, 2H), 1.52-1.28 (m, 10H). ${ }^{13} \mathrm{C}-\mathrm{NMR}$ (75 MHz, Chloroform-d) $\delta 169.73(\mathrm{C}=\mathrm{O}), 164.56(\mathrm{C}=\mathrm{O}), 150.68,133.80,131.75,126.03$, $123.83,123.49,65.29,51.47,29.37,29.17,29.09,28.85,28.65$, $26.70,25.94,21.10$.

12-azidododecyl 2-acetoxybenzoate (2h): light yellow oil, 91\%, ${ }^{1} \mathrm{H}-\mathrm{NMR}$ (300 MHz, Chloroform-d) $\delta 8.07$ (dd, $J=7.8$, $1.8 \mathrm{~Hz}, 1 \mathrm{H}), 7.60(\mathrm{td}, J=7.8,1.8 \mathrm{~Hz}, 1 \mathrm{H}), 7.36(\mathrm{td}, J=8.1,1.2 \mathrm{~Hz}$, $1 \mathrm{H}), 7.15(\mathrm{dd}, J=8.1,1.2 \mathrm{~Hz}, 1 \mathrm{H}), 4.31$ (t, $J=6.8 \mathrm{~Hz}, 2 \mathrm{H}), 3.30$ $(\mathrm{t}, J=7.0 \mathrm{~Hz}, 2 \mathrm{H}), 2.40(\mathrm{~s}, 3 \mathrm{H}), 1.85-1.71(\mathrm{~m}, 2 \mathrm{H}), 1.70-1.57$ (m, 2H). 1.52-1.25 (m, 16). ${ }^{13} \mathrm{C}-\mathrm{NMR}$ (75 MHz, Chloroform-d) $\delta 169.73(\mathrm{C}=\mathrm{O}), 164.58(\mathrm{C}=\mathrm{O}), 150.67,133.78,131.77,126.03$, 123.83, 123.51, 65.35, 51.51, 29.53 (x3), 29.50, 29.29, 29.18, $28.87,28.67,26.74,25.99,21.10$.

\section{Synthesis of propargyl-genistein (3)}

The intermediate $\mathbf{3}$ was synthesized following a procedure described in the literature (Ding et al., 2019): Genistein (1 mmol), DIPEA (2.5 mmol), and DMF $(5 \mathrm{ml})$ were placed in a $10 \mathrm{ml}$ flat-bottomed flask equipped with a magnetic stirring bar. The mixture was stirred for a period of 1 hour. Then, a mixture of propargyl bromide $(1.5 \mathrm{mmol}), \mathrm{KI}(0.1 \mathrm{mmol})$, and DMF $(1 \mathrm{ml})$ was added slowly to the reaction mixture and stirred for 24 hours. Water was added to reaction mixture and then it was extracted with ethyl acetate. Organic phase was dried on anhydrous sodium sulfate, filtered, concentrated under reduced pressure on a rotatory evaporator and the residue was purified by column chromatography over silica gel eluting with a mixture of hexane:ethyl acetate (ratio $85: 15)$, to obtain compound 3 in $60 \%$ yield.

${ }^{1} \mathrm{H}-\mathrm{NMR}$ (300 MHz, Dimethyl sulfoxide (DMSO-d6) $\delta$ $12.97(\mathrm{~s}, 1 \mathrm{H}), 9.63(\mathrm{~s}, 25 \mathrm{H}), 8.43(\mathrm{~s}, 1 \mathrm{H}), 7.39(\mathrm{~d}, J=8.6 \mathrm{~Hz}$, $2 \mathrm{H}), 6.83(\mathrm{~d}, J=8.6 \mathrm{~Hz}, 2 \mathrm{H}), 6.72(\mathrm{~d}, J=2.2 \mathrm{~Hz}, 1 \mathrm{H}), 6.47(\mathrm{~d}$, $J=2.3 \mathrm{~Hz}, 1 \mathrm{H}), 4.96(\mathrm{~d}, J=2.3 \mathrm{~Hz}, 2 \mathrm{H}), 3.69(\mathrm{t}, J=2.2 \mathrm{~Hz}$, 1H). ${ }^{13} \mathrm{C}-\mathrm{NMR}$ (75 MHz, Chloroform-d) $\delta 181.04(\mathrm{C}=\mathrm{O}), 163.22$, $162.09,157.79,157.19,152.99(\mathrm{O}-\underline{\mathrm{CH}}=\mathrm{C}$, chromone), 130.10, $123.92,123.88,121.56,115.47,106.56,98.83,93.30,93.25$, 77.22, 76.62 (alkyne), 56.14.

General procedure for the synthesis of ASA-Genistein hybrids $(\mathbf{4 a}-\mathbf{h})$

Hybrids $\mathbf{4 a - h}$ were synthesized following a procedure described in the literature (Shinde et al., 2019): Propargylgenistein (1 mmol), ASA-alkylazides (2a-h) $(1 \mathrm{mmol})$, and DMF $(5 \mathrm{ml})$ were placed in a $10 \mathrm{ml}$ flat-bottomed flask. The mixture was sonicated for 5 minutes to $40^{\circ} \mathrm{C}$. Then, a mixture of ascorbic acid $(0.5 \mathrm{mmol})$, copper acetate $(0.5 \mathrm{mmol})$, DMF $(1 \mathrm{ml})$, and water $(1 \mathrm{ml})$ was added to the reaction mixture and sonicated for 1 hour to $40^{\circ} \mathrm{C}$. Solution of $\mathrm{HCl} 10 \%$ was added to reaction mixture and then it was extracted with ethyl acetate. Organic phase was dried on anhydrous sodium sulfate, filtered, concentrated under reduced pressure on a rotatory evaporator and the residue was submitted to crystallization (MeOH: $\mathrm{H}_{2} \mathrm{O}, 1: 1$ ratio). Finally, the solid obtained was purified by preparative chromatography over silica gel eluting with a mixture of hexane/ethyl acetate of different ratios, to obtain compounds $\mathbf{4 a}-\mathbf{h}$ in yields ranging between $43 \%$ and $94 \%$.

3-(4-(((5-hydroxy-3-(4-hydroxyphenyl)-4-oxo-4H-chromen-7yl)oxy)methyl)-1H-1,2,3-triazol-1-yl)ethyl 2-acetoxybenzoate (4a)

Yield 94\%; pale yellow oil; ${ }^{1} \mathrm{H}-\mathrm{NMR}(300 \mathrm{MHz}$, DMSO-d6) $\delta 9.61(\mathrm{~s}, \mathrm{OH}), 8.40(\mathrm{~s}, 1 \mathrm{H}$, triazolyl), $8.37(\mathrm{~s}, 1 \mathrm{H}$, -O-CH=C-, chromone), 7.83 (d, $J=7.8 \mathrm{~Hz}, 1 \mathrm{H}), 7.67$ (t, $J=$ $8.1 \mathrm{~Hz}, 1 \mathrm{H}), 7.40(\mathrm{~d}, J=8.2 \mathrm{~Hz}, 2 \mathrm{H}), 7.37$ (t, $J=7.8 \mathrm{~Hz}, 1 \mathrm{H})$, $7.22(\mathrm{~d}, J=8.1 \mathrm{~Hz}, 1 \mathrm{H}), 6.84(\mathrm{~d}, J=8.2 \mathrm{~Hz}, 2 \mathrm{H}), 6.78(\mathrm{~d}, J=$ $1.5 \mathrm{~Hz}, 1 \mathrm{H}), 6.49(\mathrm{~d}, J=1.5 \mathrm{~Hz}, 1 \mathrm{H}), 5.30(\mathrm{~s}, 2 \mathrm{H}), 4.80(\mathrm{t}, J=$ $4.9 \mathrm{~Hz}, 2 \mathrm{H}), 4.66$ (t, $J=4.9 \mathrm{~Hz}, 2 \mathrm{H}), 2.22(\mathrm{~s}, 3 \mathrm{H}) .{ }^{13} \mathrm{C}-\mathrm{NMR}$ (75 MHz, DMSO-d6) $\delta 180.87(\mathrm{C}=\mathrm{O}$ chromone), $169.56(\mathrm{C}=\mathrm{O})$ $164.28(\mathrm{C}=\mathrm{O}), 163.82,162.17,157.94,157.85,154.92,150.61$ (-O-CH=C-,chromone), 134.99 (triazolyl), 131.97, 131.60, $130.63(\mathrm{x} 2), 126.68,125.82,124.53,122.98,122.85,121.48$ (triazolyl), $115.54(\mathrm{x} 2), 106.04,99.06,93.59,63.70,62.26$, 49.05, 21.10. HRMS-ESI (m/z): $558.1509[\mathrm{M}+\mathrm{H}]^{+}$, calcd. for $\mathrm{C}_{29} \mathrm{H}_{24} \mathrm{~N}_{3} \mathrm{O}_{9}[\mathrm{M}+\mathrm{H}]^{+}$558.1507. HRMS-ESI $m / z$ (\% rel. abd.): $558.1509\left(\mathrm{M}^{+}, 4\right), 516.1403$ (100), 396.1189 (31), 368.1127 (7), 307.0603 (12), 271.0602 (73).

3-(4-(((5-hydroxy-3-(4-hydroxyphenyl)-4-oxo-4H-chromen-7-yl) oxy)methyl)-1H-1,2,3-triazol-1-yl)propyl 2-acetoxybenzoate (4b)

Yield $80 \%$; pale yellow oil; ${ }^{1} \mathrm{H}-\mathrm{NMR}(300 \mathrm{MHz}$, DMSO-d6) $\delta 9.61(\mathrm{~s}, \mathrm{OH}), 8.42(\mathrm{~s}, 1 \mathrm{H}$, triazolyl), $8.34(\mathrm{~s}, 1 \mathrm{H}$, -O-CH=C-, chromone), 7.94 (dd, $J=7.8,1.6 \mathrm{~Hz}, 1 \mathrm{H}), 7.69$ (td, $J$ $=8.0,1.6 \mathrm{~Hz}, 1 \mathrm{H}), 7.43(\mathrm{~d}, J=7.8 \mathrm{~Hz}, 1 \mathrm{H}), 7.40(\mathrm{~d}, J=8.6 \mathrm{~Hz}$, $2 \mathrm{H}), 7.25(\mathrm{~d}, J=8.0 \mathrm{~Hz}, 1 \mathrm{H}), 6.84(\mathrm{~d}, J=8.6 \mathrm{~Hz}, 2 \mathrm{H}), 6.80(\mathrm{~d}, J$ $=2.2 \mathrm{~Hz}, 1 \mathrm{H}), 6.50(\mathrm{~d}, J=2.2 \mathrm{~Hz}, 1 \mathrm{H}), 5.28(\mathrm{~s}, 2 \mathrm{H}), 4.55(\mathrm{t}, J=$ $6.9 \mathrm{~Hz}, 1 \mathrm{H}), 4.25$ (t, $J=6.2 \mathrm{~Hz}, 2 \mathrm{H}), 2.52-2.50$ (m, 2H), 2.29 (s, $3 \mathrm{H}) .{ }^{13} \mathrm{C}-\mathrm{NMR}(75 \mathrm{MHz}, \mathrm{DMSO}-\mathrm{d} 6) \delta 180.89$ (C=O chromone), $169.64(\mathrm{C}=\mathrm{O}), 164.32(\mathrm{C}=\mathrm{O}), 164.26,162.19,157.94,157.88$, 154.95, 150.52 (-O-CH=C-,chromone), 142.30 (triazolyl), 134.84, $131.79,130.64$ (x2), 126.72, 125.52, 124.47, 123.29, 122.99, 121.48 (triazolyl), 115.54 (x2), 106.05, 99.06, 93.62, 62.53, 62.31, 47.08, 29.29, 21.24. HRMS-ESI $(\mathrm{m} / \mathrm{z}): 572.1664[\mathrm{M}+\mathrm{H}]^{+}$calcd. for $\mathrm{C}_{30} \mathrm{H}_{26} \mathrm{~N}_{3} \mathrm{O}_{9}[\mathrm{M}+\mathrm{H}]^{+}$572.1663. HRMS-ESI $m / z$ (\% rel. abd.): $572.1664\left(\mathrm{M}^{+}, 2\right), 530.1562$ (100), 410.1347 (67), 307.0599 (8), 271.0603 (49), 232.0971 (38).

3-(4-(((5-hydroxy-3-(4-hydroxyphenyl)-4-oxo-4H-chromen-7-yl) oxy)methyl)-1H-1,2,3-triazol-1-yl)butyl 2-acetoxybenzoate (4c)

Yield 70\%; pale yellow oil; ${ }^{1} \mathrm{H}-\mathrm{NMR}(300 \mathrm{MHz}$, DMSO-d6) $\delta 8.41(\mathrm{~s}, 1 \mathrm{H}$, triazolyl), $8.31(\mathrm{~s}, 1 \mathrm{H},-\mathrm{O}-\mathrm{CH}=\mathrm{C}-$, chromone), 7.94 (dd, $J=7.8,1.6 \mathrm{~Hz}, 1 \mathrm{H}), 7.68$ (td, $J=8.1,1.6$ $\mathrm{Hz}, 1 \mathrm{H}), 7.42$ (d, $J=7.8 \mathrm{~Hz}, 1 \mathrm{H}), 7.40$ (d, $J=8.6 \mathrm{~Hz}, 2 \mathrm{H}), 7.23$ $(\mathrm{d}, J=8.1 \mathrm{~Hz}, 1 \mathrm{H}), 6.84(\mathrm{~d}, J=8.6 \mathrm{~Hz}, 2 \mathrm{H}), 6.80(\mathrm{~d}, J=2.2 \mathrm{~Hz}$, $1 \mathrm{H}), 6.51(\mathrm{~d}, J=2.2 \mathrm{~Hz}, 1 \mathrm{H}), 5.28(\mathrm{~s}, 2 \mathrm{H}), 4.46(\mathrm{t}, J=7.1 \mathrm{~Hz}, 2 \mathrm{H})$, $4.25(\mathrm{t}, J=6.5 \mathrm{~Hz}, 2 \mathrm{H}), 2.25(\mathrm{~s}, 3 \mathrm{H}), 1.99-1.91(\mathrm{~m}, 2 \mathrm{H}), 1.70$ $1.64(\mathrm{~m}, 2 \mathrm{H}) .{ }^{13} \mathrm{C}-\mathrm{NMR}(75 \mathrm{MHz}, \mathrm{DMSO}-\mathrm{d} 6) \delta 180.88(\mathrm{C}=\mathrm{O}$ chromone), $169.59(\mathrm{C}=\mathrm{O}), 164.47(\mathrm{C}=\mathrm{O}), 164.33$, 162.18, 157.94, $157.87,154.93,150.40,142.22$ (triazolyl), 134.73, 131.66, 130.63 (x2), 126.76, 125.38, 124.48, 123.57, 122.99, 121.48 (triazolyl), 115.54 (x2), 106.04, 99.07, 93.63, 64.66, 62.33, 49.47, 26.80, 25.66, 21.20. HRMS-ESI $(\mathrm{m} / \mathrm{z}): 586.1820[\mathrm{M}+\mathrm{H}]^{+}$calcd. for $\mathrm{C}_{31} \mathrm{H}_{28} \mathrm{~N}_{3} \mathrm{O}_{9}[\mathrm{M}+\mathrm{H}]^{+}$586.1827. HRMS-ESI $m / z$ (\% rel. abd.): 
$586.1820\left(\mathrm{M}^{+}, 1\right), 544.1719(100), 424.1506$ (60), 406.1399 (29), 378.1335 (4), 307.0603 (5).

3-(4-(((5-hydroxy-3-(4-hydroxyphenyl)-4-oxo-4H-chromen-7-yl) oxy)methyl)-1H-1,2,3-triazol-1-yl)pentyl 2-acetoxybenzoate (4d)

Yield 86\%; pale yellow oil; ${ }^{1} \mathrm{H}-\mathrm{NMR}(300 \mathrm{MHz}$, Chloroform-d) $\delta 12.85$ (s, OH), 7.99 (d, $J=8.0 \mathrm{~Hz}, 1 \mathrm{H}), 7.84$ (s, $1 \mathrm{H}$, triazolyl), 7.65 (s, $1 \mathrm{H},-\mathrm{O}-\mathrm{CH}=\mathrm{C}-$, chromone), 7.58 (t, $J=7.6$ $\mathrm{Hz}, 1 \mathrm{H}), 7.35$ (d, $J=8.1 \mathrm{~Hz}, 2 \mathrm{H}), 7.33$ (d, $J=7.6 \mathrm{~Hz}, 1 \mathrm{H}), 7.12$ $(\mathrm{d}, J=8.0 \mathrm{~Hz}, 1 \mathrm{H}), 6.90(\mathrm{~d}, J=8.1 \mathrm{~Hz}, 2 \mathrm{H}), 6.49(\mathrm{~d}, J=2.0 \mathrm{~Hz}$, $1 \mathrm{H}), 6.41(\mathrm{~d}, J=2.0 \mathrm{~Hz}, 1 \mathrm{H}), 5.21(\mathrm{~s}, 2 \mathrm{H}), 4.41(\mathrm{t}, J=7.1 \mathrm{~Hz}, 2 \mathrm{H})$, $4.30(\mathrm{t}, J=6.5 \mathrm{~Hz}, 2 \mathrm{H}), 2.36(\mathrm{~s}, 3 \mathrm{H}), 2.05-1.98(\mathrm{~m}, 2 \mathrm{H}), 1.84-1.78$ (m, 2H), 1.52-1.45 (m, 2H). ${ }^{13} \mathrm{C}-\mathrm{NMR}(75 \mathrm{MHz}$, Chloroform-d) $\delta 180.92(\mathrm{C}=\mathrm{O}$ chromone $), 169.94(\mathrm{C}=\mathrm{O}), 164.49(\mathrm{C}=\mathrm{O}), 163.93$, $162.58,157.86,156.57,152.87,150.68,142.85$ (triazolyl), 134.05, $131.65,130.20(\mathrm{x} 2), 126.16,123.84,123.72,123.19,122.38$ (triazolyl), 115.73 (x2), 106.56, 98.97, 93.07, 64.52, 63.73, 62.11, $50.38,29.79,28.03,22.97,21.14$. HRMS-ESI $(\mathrm{m} / \mathrm{z}): 600.1984$ $[\mathrm{M}+\mathrm{H}]^{+}$calcd. for $\mathrm{C}_{32} \mathrm{H}_{30} \mathrm{~N}_{3} \mathrm{O}_{9}[\mathrm{M}+\mathrm{H}]^{+}$600.1976. HRMS-ESI $\mathrm{m} / z$ (\% rel. abd.): $600.1984\left(\mathrm{M}^{+}, 1\right), 558.1881$ (100), 438.1665 (58), 302.1384 (15), 271.0601 (28), 140.1076 (12).

3-(4-(((5-hydroxy-3-(4-hydroxyphenyl)-4-oxo-4H-chromen-7-yl) oxy)methyl)-1H-1,2,3-triazol-1-yl)hexyl 2-acetoxybenzoate (4e)

Yield 43\%; pale yellow oil; ${ }^{1} \mathrm{H}-\mathrm{NMR}$ (300 MHz, Chloroform-d) $\delta 12.83(\mathrm{~s}, \mathrm{OH}), 7.99(\mathrm{dd}, J=7.8,1.5 \mathrm{~Hz}, 1 \mathrm{H})$, 7.81 (s, $1 \mathrm{H}$, triazolyl), 7.63 (s, $1 \mathrm{H},-\mathrm{O}-\mathrm{CH}=\mathrm{C}-$, chromone), 7.55 $(\mathrm{td}, J=7.8,1.5 \mathrm{~Hz}, 1 \mathrm{H}), 7.32(\mathrm{~d}, J=8.0 \mathrm{~Hz}, 2 \mathrm{H}), 7.30(\mathrm{~d}, J=7.8$ $\mathrm{Hz}, 1 \mathrm{H}), 7.10(\mathrm{~d}, \mathrm{~J}=8.0 \mathrm{~Hz}, 1 \mathrm{H}), 6.88(\mathrm{~d}, J=8.0 \mathrm{~Hz}, 2 \mathrm{H}), 6.48(\mathrm{~d}$, $J=2.2 \mathrm{~Hz}, 1 \mathrm{H}), 6.39(\mathrm{~d}, J=2.2 \mathrm{~Hz}, 1 \mathrm{H}), 5.21(\mathrm{~s}, 2 \mathrm{H}), 4.37(\mathrm{t}, J=$ $7.2 \mathrm{~Hz}, 2 \mathrm{H}), 4.26$ (t, $J=6.6 \mathrm{~Hz}, 2 \mathrm{H}), 2.34$ (s, 3H), 1.99-1.91 (m, $2 \mathrm{H}), 1.80-1.70(\mathrm{~m}, 2 \mathrm{H}), 1.51-1.43(\mathrm{~m}, 2 \mathrm{H}), 1.43-1.36(\mathrm{~m}, 2 \mathrm{H})$. ${ }^{13} \mathrm{C}-\mathrm{NMR}$ (75 MHz, Chloroform-d) $\delta 180.91$ ( $\mathrm{C}=\mathrm{O}$ chromone), $169.87(\mathrm{C}=\mathrm{O}), 164.54(\mathrm{C}=\mathrm{O}), 163.96,156.27,162.64,157.89$, $152.86,150.67,143.12$ (triazolyl), 133.96, 131.70, 130.25 (x2), $126.12,123.84,123.73,123.30,122.78,122.57$ (triazolyl), 115.70 (x2) $106.59,99.01,93.11,65.90,64.87,62.24,30.15,28.46$, 26.15, 25.44, 21.13. HRMS-ESI $(\mathrm{m} / \mathrm{z}): 614.2133[\mathrm{M}+\mathrm{H}]^{+}$calcd. for $\mathrm{C}_{33} \mathrm{H}_{32} \mathrm{~N}_{3} \mathrm{O}_{9}[\mathrm{M}+\mathrm{H}]^{+}$614.2133. HRMS-ESI $m / z$ (\% rel. abd.): $614.2133\left(\mathrm{M}^{+}, 1\right), 572.2027$ (100), 452.1817 (48), 424.1754 (3), 274.1437 (36), 154.1225 (19).

3-(4-(((5-hydroxy-3-(4-hydroxyphenyl)-4-oxo-4H-chromen-7-yl) oxy)methyl)-1H-1,2,3-triazol-1-yl)octyl 2-acetoxybenzoate (4f)

Yield 60\%; pale yellow oil; ${ }^{1} \mathrm{H}-\mathrm{NMR}(300 \mathrm{MHz}$, Chloroform-d) $\delta 12.84(\mathrm{~s}, \mathrm{OH}), 8.00(\mathrm{dd}, J=8.0,1.0 \mathrm{~Hz}, 1 \mathrm{H})$, $7.83(\mathrm{~s}, 1 \mathrm{H}$, triazolyl), $7.63(\mathrm{~s}, 1 \mathrm{H},-\mathrm{O}-\mathrm{CH}=\mathrm{C}-$, chromone $), 7.58$ $-7.53(\mathrm{~m}, 1 \mathrm{H}), 7.34(\mathrm{~d}, J=8.0 \mathrm{~Hz}, 2 \mathrm{H}), 7.31(\mathrm{t}, J=7.6 \mathrm{~Hz}, 1 \mathrm{H})$, $7.10(\mathrm{~d}, J=8.0 \mathrm{~Hz}, 1 \mathrm{H}), 6.92-6.86(\mathrm{~m}, 2 \mathrm{H}), 6.50(\mathrm{~d}, 1.1 \mathrm{~Hz}, 1 \mathrm{H})$, $6.42(\mathrm{~d}, J=2.0 \mathrm{~Hz}, 1 \mathrm{H}), 5.24(\mathrm{~s}, 2 \mathrm{H}), 4.36(\mathrm{t}, J=7.2 \mathrm{~Hz}, 2 \mathrm{H}), 4.25$ (t, $J=6.7 \mathrm{~Hz}, 2 \mathrm{H}), 2.34$ (s, 3H), 1.95-1.89 (m, 2H), 1.75-1.68 $(\mathrm{m}, 2 \mathrm{H}), 1.44-1.29(\mathrm{~m}, 6 \mathrm{H}), 1.28-1.22(\mathrm{~m}, 2 \mathrm{H}) .{ }^{13} \mathrm{C}-\mathrm{NMR}(75$ $\mathrm{MHz}$, Chloroform-d) $\delta 180.92(\mathrm{C}=\mathrm{O}$ chromone $), 169.89(\mathrm{C}=\mathrm{O})$, $164.59(\mathrm{C}=\mathrm{O}), 163.94,162.63,157.89,156.47,152.84,150.66$, 142.84 (triazolyl), 133.88, 131.74, 130.21 (x2), 126.10, 123.83, 123.75, 123.40, 122.98, 122.47 (triazolyl), 115.73 (x2), 106.59, $99.02,93.12,65.20,62.24,50.63,30.23,29.74,28.87,28.58$, 26.41, 25.83, 21.13. HRMS-ESI $(\mathrm{m} / \mathrm{z}): 642.2447[\mathrm{M}+\mathrm{H}]^{+}$calcd. for $\mathrm{C}_{35} \mathrm{H}_{36} \mathrm{~N}_{3} \mathrm{O}_{9}[\mathrm{M}+\mathrm{H}]^{+}$642.2446. HRMS-ESI $m / z$ (\% rel. abd.): $642.2447\left(\mathrm{M}^{+}, 2\right), 600.2342$ (100), 480.2132 (51), 330.1813 (29), 302.1752 (47), 271.0602 (15).

3-(4-(((5-hydroxy-3-(4-hydroxyphenyl)-4-oxo-4H-chromen-7-yl) oxy)methyl)-1H-1,2,3-triazol-1-yl)nonyl 2-acetoxybenzoate (4g)

Yield 50\%; pale yellow oil; ${ }^{1} \mathrm{H}-\mathrm{NMR}(300 \mathrm{MHz}$, Chloroform-d) $\delta 12.86$ (s, OH), 8.03 (dd, $J=7.8,1.3 \mathrm{~Hz}, 1 \mathrm{H}), 7.85$ (s, 1H, triazolyl), 7.65 (s, 1H, -O-CH=C-, chromone), 7.59-7.55 $(\mathrm{m}, 1 \mathrm{H}), 7.36(\mathrm{~d}, J=8.2 \mathrm{~Hz}, 2 \mathrm{H}), 7.33(\mathrm{t}, J=7.8 \mathrm{~Hz}, 1 \mathrm{H}), 7.12$ $(\mathrm{d}, J=8.1 \mathrm{~Hz}, 1 \mathrm{H}), 6.91(\mathrm{~d}, J=8.2 \mathrm{~Hz}, 2 \mathrm{H}), 6.52(\mathrm{~d}, J=2.1 \mathrm{~Hz}$, $1 \mathrm{H}), 6.44(\mathrm{~d}, J=2.1 \mathrm{~Hz}, 1 \mathrm{H}), 5.27(\mathrm{~s}, 2 \mathrm{H}), 4.38(\mathrm{t}, J=7.2 \mathrm{~Hz}$, $2 \mathrm{H}), 4.27$ (t, $J=6.7 \mathrm{~Hz}, 2 \mathrm{H}), 2.37(\mathrm{~s}, 3 \mathrm{H}), 1.98-1.91(\mathrm{~m}, 2 \mathrm{H})$, $1.78-1.69(\mathrm{~m}, 2 \mathrm{H}), 1.46-1.26(\mathrm{~m}, 10 \mathrm{H}) .{ }^{13} \mathrm{C}-\mathrm{NMR}(75 \mathrm{MHz}$, Chloroform-d) $\delta 180.92(\mathrm{C}=\mathrm{O}$ chromone), $169.92(\mathrm{C}=\mathrm{O}), 164.61$ $(\mathrm{C}=\mathrm{O}), 163.92,162.60,157.88,156.56,152.85,150.65,142.82$ (triazolyl), 133.87, 131.75, 130.19 (x2), 126.09, 123.82, 123.75, 123.41, 123.00, 122.38 (triazolyl), 115.75 (x2), 106.58, 99.02, $93.11,65.28,62.20,50.66,30.25,29.25,29.11,28.90,28.61$, 26.45, 25.89, 21.13. HRMS-ESI $(\mathrm{m} / \mathrm{z}): 656.2603[\mathrm{M}+\mathrm{H}]^{+}$calcd. for $\mathrm{C}_{36} \mathrm{H}_{38} \mathrm{~N}_{3} \mathrm{O}_{9}[\mathrm{M}+\mathrm{H}]^{+}$656.2602. HRMS-ESI $m / z$ (\% rel. abd.): $656.2603\left(\mathrm{M}^{+}, 2\right), 614.2494$ (100), 494.2283 (49), 344.1969 (27), 316.1907 (46), 271.0600 (15).

3-(4-(((5-hydroxy-3-(4-hydroxyphenyl)-4-oxo-4H-chromen-7-yl) oxy)methyl)-1H-1,2,3-triazol-1-yl)dodecyl 2-acetoxybenzoate (4h)

Yield 77\%; pale yellow oil; ${ }^{1} \mathrm{H}-\mathrm{NMR}(300 \mathrm{MHz}$, Chloroform-d) $\delta 12.84(\mathrm{~s}, \mathrm{OH}), 8.01$ (d, $J=8.1 \mathrm{~Hz}, 1 \mathrm{H}), 7.82$ (s, 1H, triazolyl), $7.64(\mathrm{~s}, 1 \mathrm{H},-\mathrm{O}-\mathrm{CH}=\mathrm{C}-$, chromone $), 7.55(\mathrm{t}$, $J=7.6 \mathrm{~Hz}, 1 \mathrm{H}), 7.34(\mathrm{~d}, J=7.8 \mathrm{~Hz}, 2 \mathrm{H}), 7.30(\mathrm{t}, J=7.6 \mathrm{~Hz}$, $1 \mathrm{H}), 7.09(\mathrm{~d}, J=8.1 \mathrm{~Hz}, 1 \mathrm{H}), 6.88(\mathrm{~m}, 2 \mathrm{H}), 6.49(\mathrm{~d}, J=1.7 \mathrm{~Hz}$, $1 \mathrm{H}), 6.41(\mathrm{~d}, J=1.7 \mathrm{~Hz}, 1 \mathrm{H}), 5.25(\mathrm{~s}, 2 \mathrm{H}), 4.36(\mathrm{t}, J=7.3 \mathrm{~Hz}$, 2H), 4.25 (t, $J=6.8 \mathrm{~Hz}, 2 \mathrm{H}), 2.34(\mathrm{~s}, 3 \mathrm{H}), 1.95-1.98(\mathrm{~m}, 2 \mathrm{H})$, 1.75-1.68 (m, 4H), $1.43-1.36(\mathrm{~m}, 2 \mathrm{H}), 1.35-1.21(\mathrm{~m}, 12 \mathrm{H})$. ${ }^{13} \mathrm{C}-\mathrm{NMR}$ (75 MHz, Chloroform-d) $\delta 180.93$ (C=O chromone), $169.91(\mathrm{C}=\mathrm{O}), 164.66(\mathrm{C}=\mathrm{O}), 163.92,162.61,157.88,156.54$, $152.86,150.63,142.40$ (triazolyl), 133.85, 131.77, 130.18 (x2), $126.09,123.82,123.75,123.45,123.00,122.38$ (triazolyl), 115.76 (x2), 106.59, 99.05, 93.11, 65.40, 62.23, 50.77, 30.26, 29.51 (x3), 29.39, 29.27, 29.00, 28.65, 26.51, 25.96, 21.13. HRMS-ESI $(\mathrm{m} / z): 698.3072[\mathrm{M}+\mathrm{H}]^{+}$calcd. for $\mathrm{C}_{39} \mathrm{H}_{44} \mathrm{~N}_{3} \mathrm{O}_{9}[\mathrm{M}$ $+\mathrm{H}]^{+}$698.3072. HRMS-ESI $m / z$ (\% rel. abd.): $698.3072(\mathrm{M}+, 5)$, 656.2963 (100), 536.2752 (40), 386.2437 (26), 358.2376 (41), 307.0598 (4).

\section{In vitro biological assays}

Cell lines and culture medium

Biological experiments were performed using SW480 colon cancer cells, its metastatic derivative (SW620) and normal cells (CHO-K1) obtained from The European Collection of Authenticated Cell Cultures (ECACC, England). Cells were cultured in $25-\mathrm{cm}^{2}$ flasks containing Dulbecco's Modified Eagle Medium, supplemented with $10 \%$ horse serum, $1 \%$ non-essential amino acids, and 1\% penicillin/streptomycin (Gibco Invitrogen, Carlsbad, USA). For all experiments, there was a reduction in horse serum (to $3 \%$ ) and the medium was supplemented with 
insulin $(10 \mathrm{mg} / \mathrm{ml})$, transferrin $(5 \mathrm{mg} / \mathrm{ml})$, and selenium $(5 \mathrm{mg} /$ ml) (ITS-defined medium; Gibco, Invitrogen, Carlsbad, USA) (Herrera et al., 2018).

\section{Growth inhibition (SRB)}

The growth inhibition of the new hybrids based on ASA/Genistein and the standard drug (5-fluorouracil; 5-FU) was evaluated through sulforhodamine B (SRB) assay. Briefly, cells were seeded at 20.000 cells/well in 96-well tissue culture plates. Then, these were incubated at $37^{\circ} \mathrm{C}$ and $5 \% \mathrm{CO}_{2}$. After a 24-hour adaptation period for the cells, they were treated with the vehicle $(1 \%$ of DMSO) or increasing concentrations $(0.01-300 \mu \mathrm{M})$ of the hybrids. After the treatment, it was added trichloroacetic acid (50\% $\mathrm{v} / \mathrm{v}$; MERCK) and left for 1 hour at $4{ }^{\circ} \mathrm{C}$. Cells proteins were further stained with $0.4 \%$ SRB (w/v; Sigma-Aldrich, United States), and washed with $1 \%$ acetic acid. After air-drying, Tris-base $(10 \mathrm{mM})$ was added to solubilize protein bound stain. A microplate reader (Mindray MR-96A) was used to read the absorbance at $492 \mathrm{~nm}$ (Pérez et al., 2014). All experiments were performed in triplicate.

\section{Statistical analysis}

All experiments were performed at least in triplicate. Data are reported as mean $\pm \mathrm{SE}$ (standard error). Statistical analysis to demonstrate the differences were evaluated by oneway ANOVA followed by the Dunnett's test. The results with $p$ values lower than 0.05 were considered significant. GraphPad Prism version 8.0.1 for Windows was used to analyze all data (Graph Pad Software, San Diego, California, USA).

\section{Molecular docking studies}

\section{Protein structure and setup}

To propose a plausible mode of action for the active hybrids $\mathbf{4 a}$ and $\mathbf{4 c}$, in this work, five apoptotic proteins and one pro-inflammatory protein were selected, which are closely associated with activation and progression of CRC. The crystal structures of Plk1 (PDB: 2OWB, ATP-binding site), Plk1 (PDB 4X9R, PBD domain), Bcl-2 (PDB: 4MAN), BAX (PDB: 4S0O), caspase-3 (PDB: 5I9B), TNF- $\alpha$ (PDB: 2AZ5), and COX-2 (PDB: 3LN1) were retrieved from the Protein Data Bank. Discovery Studio Visualizer was used to removed crystallographic water molecules and metal-ions in the 3D structure. AutoDockTools was used for parameterizing the structures of the selected proteins (Morris et al., 2009). Overall, to polar side chains hydrogens were added to facilitate the formation of bonds, and the assignment of appropriate atomic partial charges to the ligand was essential to obtain meaningful results from electrostatic calculations (Kollman and Gasteiger). AutoDock was used to save the resulting protein file in Protein Data Bank, Partial Charge (Q), \& Atom Type (T) (PDBQT) format.

\section{Ligand dataset preparation and optimization}

Here, novel hybrids compounds $\mathbf{4 a}$ and $\mathbf{4 c}$, and six inhibitors that have been used as marketed drugs or potent drug candidates: Volasertib (Plk1 ATP-site), PHA-680626 (Plk1 ATPsite), ABT-199 (Bcl-2), ICPM (Plk1 PBD-site), a inhibitor of TNF- $\alpha$, and the anti-inflammatory drug celecoxib (COX-2). Aiming to rewrite the data files into pdb format, Discovery Studio Visualizer was used. AutoDockTools was used to parameterize the structures of the ligands. Then, full hydrogens were added, rotatable bonds were assigned, partial atomic charges computed (Kollman and Gasteiger), and the resulting structures saved to be used AutoDock. Moreover, AUTOTUTORS in AutoDockTools were used to define all probable flexible torsions of the ligand molecules in order to facilitate hybrids receptor binding during simulations (Morris et al., 1998, 2009).

\section{Docking analysis}

AutoDock Vina were used aiming to run docking simulations considering flexible ligand to a rigid target. Catalytic sites of each selected protein-target were chossen to run docking calculations. Then, the most favorable binding pose and affinity scores (in $\mathrm{kcal} / \mathrm{mol}$ ) were determinated. Assisted by AutoDock Vina 1.1.2 (Trott et al., 2010), docking calculations were accomplished into a grid box enclosing the catalytic domain of 38 $\times 38 \times 38 \AA$ with a grid spacing of $1 \AA$, and exhaustiveness of 20 . Finally, The PyMOL Molecular Graphics System 2.0 programs and DS Visualizer 2.5 (http://3dsbiovia.com/products/) were used to graphically inspect the binding docking conformations and generate the 2D ligand interaction diagrams, respectively.

\section{Theorical drug-likeness studies}

In silico drug-likeness studies along with further ADMET (absorption, distribution, metabolism, excretion, and toxicity) indices present an array of opportunities which help in accelerating the discovery of new anticancer candidates. In this scenario, for active hybrids $\mathbf{4 a}$ and $\mathbf{4 c}$, eleven key biopharmaceutical parameters were estimated by using the opensource platforms Molinspiration software (for: Topographical polar surface area (TPSA), molecular weight (MW), and rotatable bonds descriptors), the Virtual Computational Chemistry Laboratory (ALOGPS 2.1) for Log $\mathrm{P}_{\mathrm{o} / \mathrm{w}}$ descriptor, and the Pre-ADMET 2.0 cheminformatic tool for estimation of key pharmacokinetic indices such as $\operatorname{LogK}_{\mathrm{HSA}}$ (human serum albumin), apparent predicted intestinal permeability models (App. Caco-2 and MDCK), and Gastrointestinal uptake and absorption (\% GI). Note that, these important biopharmaceutical parameters govern oral exposure, as well as permeability, absorption, motion, and action of potential drug candidates. SwissADME (Daina et al., 2017), a free online software, was used to find out alerts for pan-assay interference compounds (PAINS). The OSIRIS Property Explorer (free open source) was used to evaluate the overall drug-score and the most common toxicity human parameters, such as mutagenicity, tumorigenicity, irritant effect, and possible injuries that can affect the reproductive system. Finally, MetStabOn, a powerful online server for the qualitative prediction of metabolic stability (Podlewska et al., 2018), was used to estimate the metabolic stability for the top-two hybrids $\mathbf{4 a}$ and $\mathbf{4 c}$.

\section{RESULTS AND DISCUSSION}

\section{Chemistry}

The synthesis of hybrids of this study began with the obtention of the ASA-bromoalkyl $\mathbf{1 a}-\mathbf{h}$ by means of the Williamson etherification of aspirin with $1, \omega$-dibromoalkanes $(\omega=3,4,5,8,9$, and 12$)$ with yields ranging between $32 \%$ and $57 \%$. Compounds $\mathbf{1} \mathbf{a}-\mathbf{h}$ were treated with sodium azide leading to the formation of the ASA-alkylazides $\mathbf{2} \mathbf{a}-\mathbf{h}$ in $72 \%-96 \%$ yields 

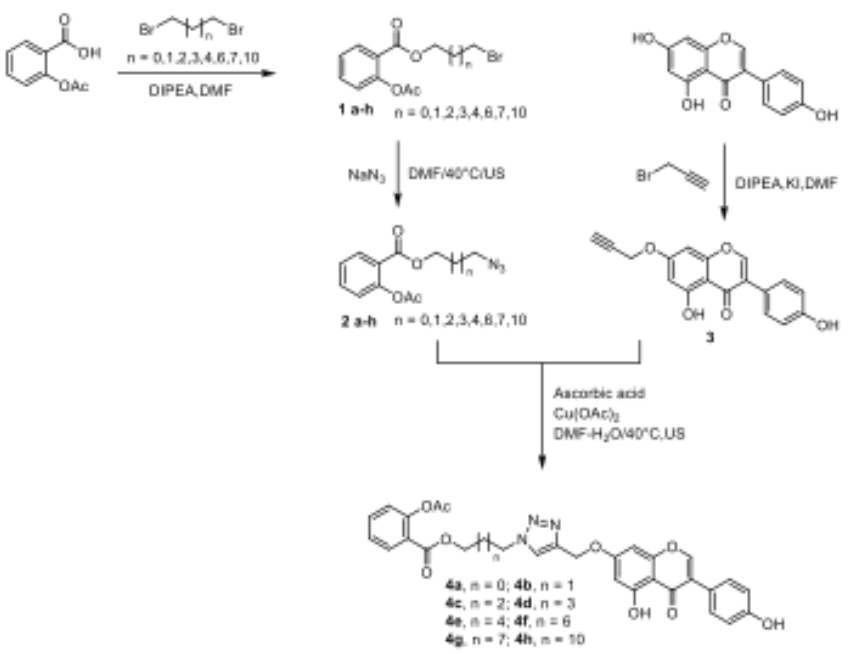

Scheme 1. Synthesis of ASA-Genistein hybrids.

(Ding et al., 2019). On the other hand, reaction of genistein with propargyl bromide led to propargyl-genistein (3) with $60 \%$ yield. Finally, click reaction between azide alkylaspirin $\mathbf{2 a - h}$ and the compound 3 led to the formation of hybrids $\mathbf{4 a}-\mathbf{h}$ in $43 \%-94 \%$ yields (Shinde et al., 2019) (Scheme 1).

The structures of all compounds have been established by a combined study of High resolution electrospray ionization mass spectrometry (HRMS-ESI) (m/z), ${ }^{1} \mathrm{H}-\mathrm{NMR}$ and ${ }^{13} \mathrm{C}-\mathrm{NMR}$ spectra. HRMS-ESI $(\mathrm{m} / \mathrm{z})$ spectra showed characteristic $[\mathrm{M}+\mathrm{H}]^{+}$ peaks corresponding to their molecular weights. The assignments of all the signals to individual $\mathrm{H}$ or $\mathrm{C}$-atoms have been performed based on typical $\delta$-values and $J$-constants. The ${ }^{1} \mathrm{H}$-NMR spectra of hybrids dissolved in DMSO or $\mathrm{CDCl}_{3}$ showed around $8.41 \mathrm{ppm}$ or $7.83 \mathrm{ppm}$, respectively, a signal corresponding to triazolyl ring. The chromone $(-\mathrm{O}-\mathrm{CH}=\mathrm{C}-)$ signal was observed around $8.30 \mathrm{ppm}$ on DMSO or $7.65 \mathrm{ppm}$ when was using $\mathrm{CDCl}_{3}$. The acetyl group of aspirin was observed around $2.25 \mathrm{ppm}$ on DMSO or $2.34 \mathrm{ppm}$ on $\mathrm{CDCl}_{3} \cdot{ }^{13} \mathrm{C}$-NMR spectra of hybrids showed around 180.8, 169.6, and 164.5 ppm signals corresponding to the carbonyl groups $(\mathrm{C}=\mathrm{O})$. The triazolyl ring exhibited signals around 142.2 and $121.4 \mathrm{ppm}$.

\section{Biological activity}

\section{Effect of ASA-Genistein hybrids SW480 and CHO-K1 cell viability}

To assess the effect of the new hybrids on cell viability, these were tested against malignant SW480 cells and normal CHO-K1 cells. As shown in Figure 2, when treated SW480 cells were microscopically observed, they exhibited a disturbed morphology after the treatment with hybrids $\mathbf{4 a}$ and $\mathbf{4 c}$, showing changes in size and shape, while the vehicle control (DMSO) showed normal and healthy shape. The severity of morphological changes increased in a time- and dose-dependent manner. Moreover, treatment with both hybrids caused a clear reduction in cell count compared to the control, suggesting an increase toward cell death or a cell cycle arrest.

The evaluated hybrids induced a time- and concentration-dependent activity. Among the results, hybrids 4a and $\mathbf{4} \mathbf{c}$ caused the highest inhibition on cell viability with increasing concentrations. This activity increased with the time, since there was not activity at 24 hours (data not shown) but it


Figure 2. Representative images of SW 480 cells 48 hours after treatment with the highest concentration evaluated ( $300 \mu \mathrm{M}$; Magnification: $40 \times)$.

\section{SW480: 48 hours post treatment}

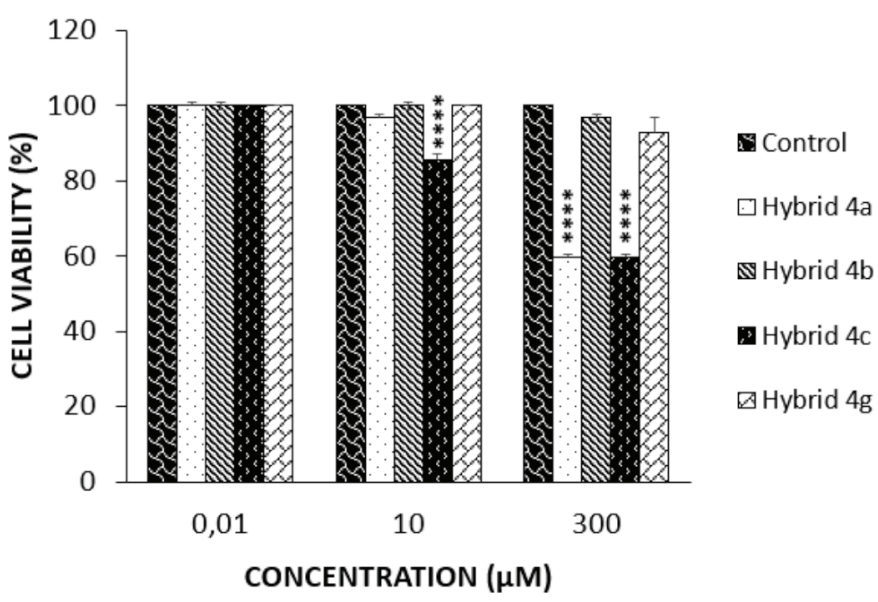

Figure 3. Effect of ASA-Genistein hybrids $(\mathbf{4 a}, \mathbf{4 b}, \mathbf{4 c}$, and $\mathbf{4 g})$ on cell viability of SW 480 cells after 48 hours treatment with $0.01,10$, and $300 \mu \mathrm{M}$. Data are presented as the mean $\pm \mathrm{SE}$ of at least three independent experiments $(* * * * p<0.0001)$. Vehicle control (DMSO 1\%) was assumed as $100 \%$ of viability.

was observed a significant activity, regarding vehicle control, after 48 hours treatment (Fig. 3). It is important to notice that these hybrid compounds increased activity and selectivity through time, which is consistent with previous reports with the parental compound aspirin where different authors also suggest that aspirin acts in a time-dependent manner (Lai et al., 2008; Satya et al., 2012; Zhang et al., 2018). This was also reflected in the $\mathrm{IC}_{50}$ values (50\% inhibitory concentration) summarized in Table 1 , where hybrids $\mathbf{4 a}$ and $\mathbf{4} \mathbf{c}$ did not display inhibition at 24 hours but after 48 hours treatment the $\mathrm{IC}_{50}$ values on the malignant SW480 cells were $449.8 \pm 25.98$ and $635.8 \pm 92.99$, respectively, besides, compound $\mathbf{4 c}$ was selective against this malignant cell line without exhibiting inhibition on healthy $\mathrm{CHO}-\mathrm{K} 1$ cells unlike the parental compound genistein $\left(\mathrm{IC}_{50}=48.44 \mu \mathrm{M} \pm 4.13\right.$ and $52.62 \mu \mathrm{M} \pm$ 8.31 for $\mathrm{CHO}-\mathrm{K} 1$ and SW480, respectively), the conventional chemotherapeutic agent, $5 \mathrm{FU}\left(\mathrm{IC}_{50}=173.2 \mu \mathrm{M} \pm 14.61\right.$ and 174.3 $\mu \mathrm{M} \pm 19.1$ for $\mathrm{CHO}-\mathrm{K} 1$ and SW480, respectively), which were even more toxic for the non-malignant cell line, and aspirin alone which did not display activity in these cells. The other synthesized hybrids (4d, 4e, 4f, and $\mathbf{4 h}$ ) did not exhibit activity in SW480 cells at the conditions evaluated. Besides, none of the compounds was active against SW620 cells (data not shown). We hypothesized that the lack of activity of these hybrid compounds could be attributed to the big size of the molecule, which makes it difficult to cross the cell membrane. These biological activities provided an approach 
Table 1. Cytotoxic effect of ASA-Genistein hybrids against SW480 and CHO-K1 cell lines at 48h.

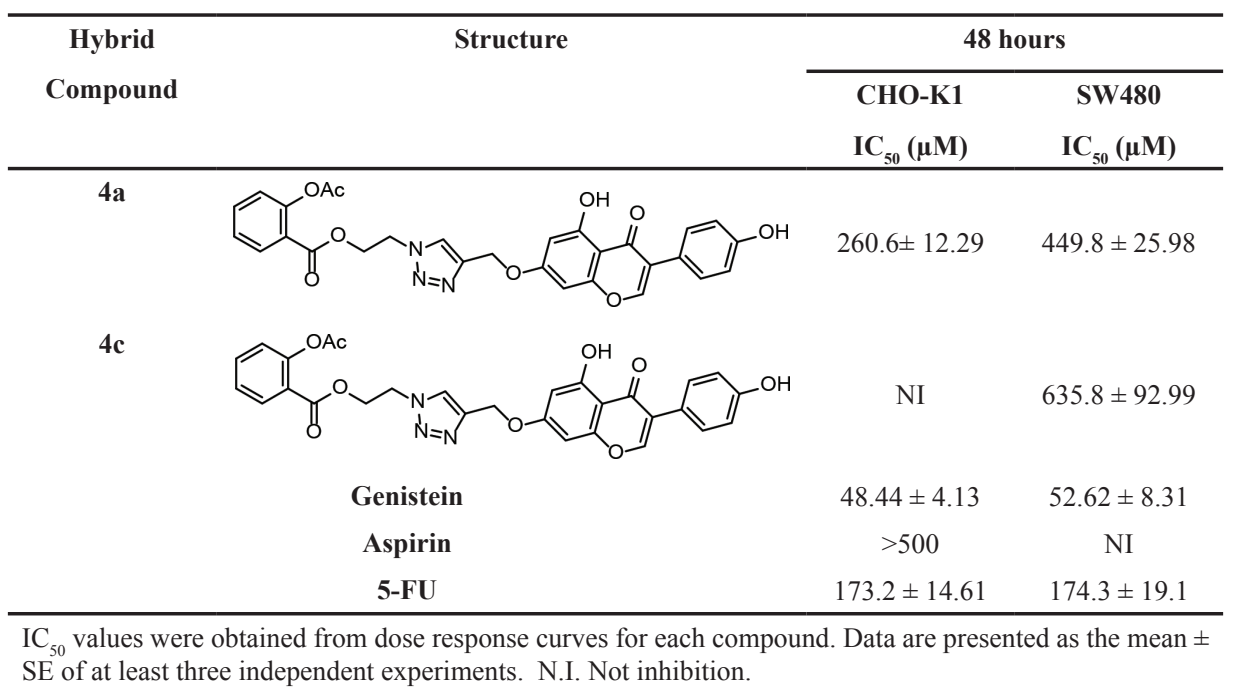

Table 2. Molecular docking scores of the top-ranked hybrids $4 \mathbf{a}$ and $\mathbf{4 c}(\mathrm{kcal} / \mathrm{mol})$ based on AutoDock Vina.

\begin{tabular}{|c|c|c|c|c|c|c|c|}
\hline \multirow[t]{2}{*}{ Target protein } & \multicolumn{7}{|c|}{ Ligands (docking score, kcal/mol) } \\
\hline & $4 a$ & $4 c$ & $\begin{array}{c}\text { Volasertib/ } \\
\text { PHA-680626 }\end{array}$ & ABT-199 & ICPM & $\begin{array}{l}\text { TNF- } \alpha \\
\text { inhibitor }\end{array}$ & Celecoxib \\
\hline Plk1 (ATP binding site) & -10.2 & -10.1 & $-9.9 /-10.5$ & - & - & - & - \\
\hline $\mathrm{Bcl}-2$ & -10.1 & -9.9 & - & -10.9 & - & - & - \\
\hline BAX & -9.8 & -9.3 & - & - & - & - & - \\
\hline Plk1 (PBD site) & -7.1 & -6.8 & - & - & -10.2 & - & - \\
\hline Caspase -3 & -6.8 & -7.0 & - & - & - & - & - \\
\hline TNF- $\alpha$ & -6.9 & -6.5 & - & - & - & -9.0 & - \\
\hline $\mathrm{COX}-2$ & -10.0 & -10.1 & - & - & - & - & -9.6 \\
\hline
\end{tabular}

to the mechanisms of action associated with the most active ASA/Genistein hybrids (4a and 4c) on SW480, which was also strengthened and expanded with the docking results, providing valuable evidence about the potential targets for these molecules in colorectal cancer.

\section{Multi-target docking studies}

Hybrids $4 a$ and $4 c$ and its interaction with the PLK-1 ATP binding domain

To provide better insights into the mechanisms by which the most active ASA/Genistein hybrids $\mathbf{4 a}$ and $\mathbf{4} \mathbf{c}$ elicit inhibitory effects on SW480 cell growth, an extensive multi-target docking and multi-site based virtual screening were performed by using multiple proteins associated with activation and progression of colorectal cancer. To achieve this purpose, authors selected five druggable proteins targets (Plk1, Bcl-2, BAX, Caspase-3, and TNF- $\alpha$ ) which play a vital role in activation and progression of CRC. Besides, considering that ASA was included in the design of the compounds, this work investigated a likely interaction of ASA/ Genistein hybrids $\mathbf{4 a}$ and $\mathbf{4} \mathbf{c}$ with the pro-inflammatory COX-2 protein which have demonstrated to induce cancer stem cell (CSC)like activity, promoting proliferation, invasion, angiogenesis, inflammation, resistance, and metastasis of cancer cells (Hashemi et al., 2019). Thus, promising $\mathbf{4 a}$ and $\mathbf{4 c}$ were docked into each catalytic domain of X-ray crystal structure of target proteins by employing grid-based ligand docking with AutoDock Vina, and affinity scores along the best binding pose (Table 2).

Bcl-2: B-cell lymphoma-2, BAX: Bcl-2 associated X protein, TNF- $\alpha$ : tumor necrosis factor alpha, Plk1: Human Pololike kinase 1, COX-2: cyclooxygenase-2. Celecoxib is a selective COX-2 inhibitor, Volasertib is an FDA-approved drug reported as potent and selective inhibitor of PLK1-ATP binding site (enzyme $\mathrm{IC}_{50}=0.87 \mathrm{nM}, \mathrm{EC}_{50}=11-37 \mathrm{nM}$ on a panel of cancer cell lines). ABT-199, Bcl-2-Navitoclax analog: potent and selective Bcl-2 inhibitor $\left(\mathrm{EC}_{50}=4 \mathrm{nM}\right)$. PHA-680626: pyrrolo-pyrazole PLK1ATP binding site inhibitor $\mathrm{IC}_{50}=\sim 0.53 \mu \mathrm{M}$. PBD: Human Polobox domain. ICPM: Imidazolium-containing phosphopeptide macrocycle, a PBD inhibitor $\left(\mathrm{IC}_{50}=15 \mathrm{nM}\right)$.

Interestingly, among these targeted proteins, hybrids $\mathbf{4 a}$ and $\mathbf{4 c}$ displayed a high-affinity binding for Plk1 (Human Pololike kinase 1) as well as for the Bcl-2/Bax system with a docking score in the range of -10.2 to $-9.3 \mathrm{kcal} / \mathrm{mol}$. Moreover, docking action of $\mathbf{4 a}$ and $\mathbf{4} \mathbf{c}$ against the pro-inflammatory COX-2 enzyme (above $10.0 \mathrm{kcal} / \mathrm{mol}$ ) suggests these compounds could act into the catalytic center of the enzyme to alter its catalytic properties. 


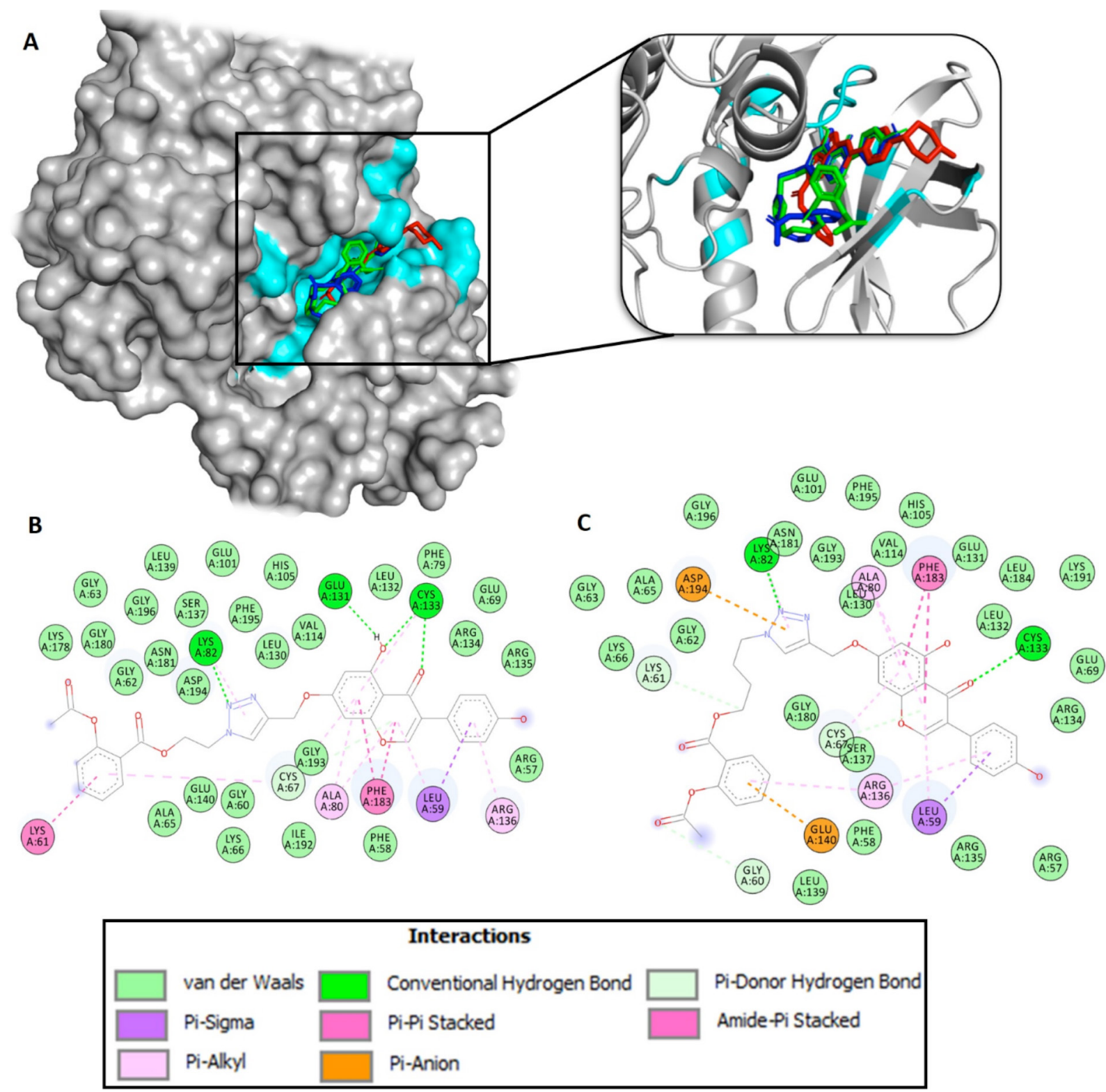

Figure 4. (A). Superposition of the best docked conformations of hybrids $4 \mathbf{a}$ (blue) and $\mathbf{4 c}$ (green), and crystallographic binding mode of inhibitor PHA-680626 (red) within the Plk1 ATP-binding domain. (B). 2D protein-ligand plot after docking procedure for 4a. (C). 2D ligand-receptor interaction diagram for $\mathbf{4 c}$. Catalytic residues on Plk1 ATP-binding domain are colored in cyan.

Therefore, authors hypothesized these facts would play a key role in SW480 growth inhibition caused by the hybrids.

Polo-like kinase 1 (Plk1) is a key regulator enzyme for cell mitosis and it is overexpressed in many cancers, particularly CRC. Targeting Plk1, specifically the Plk1-ATP binding domain, could be an effective therapeutic strategy to combat colorectal malignancies (Takahashi et al., 2003). As reported, ATP-catalytic domain of Plk1 is constituted by key binding residues which play a critical role in its biochemical function, including Arg57, Leu59, Cys67, Ala80, Lys82, Glu101, His105, Val114, Leu130, Glu131, Leu132, Cys133, Arg135, Arg136, Lys178, Asn181, and Phe183 (Kothe et al., 2007). So, docking of promising $\mathbf{4 a}$ and $\mathbf{4 c}$ into the ATP-binding pocket of Plk1 reveals several features that might be important for their cytotoxic effects displaying remarkable binding free energies of -10.2 and $-10.1 \mathrm{kcal} / \mathrm{mol}$, respectively.
As can be seen in Figure 4A, docking results revealed that these hit-compounds were well accommodated inside the binding cavity and oriented in a very similar binding mode to the X-ray pose of the inhibitor PHA-680626, besides, they exhibited key interactions with those critical residues essential for the development of Plk1 (Fig. 4B and C). Thus, lead compounds show to interact with Plk1 through three hydrogen bonds between the catalytic triad Glu131, Cys133, and Lys82 and the features of chromone and triazole. Chromone ring of $\mathbf{4 a}$ and $\mathbf{4 c}$ showed two $\pi$-contacts with Phe183 and Leu59 residues. Moreover, Asp194 (a key residue for the binding of ATP at the active site) forms one $\pi$-anion interaction with compound $\mathbf{4 c}$. In addition, numerous hydrophobic interactions were mediated by Gly62, Ala65, Leu130, Leu132, Arg134, Ser137, Glu140, Gly193, Lys178, Gly180, Asp194, and Phe195 which potentially confer stability during the binding occurrence. 

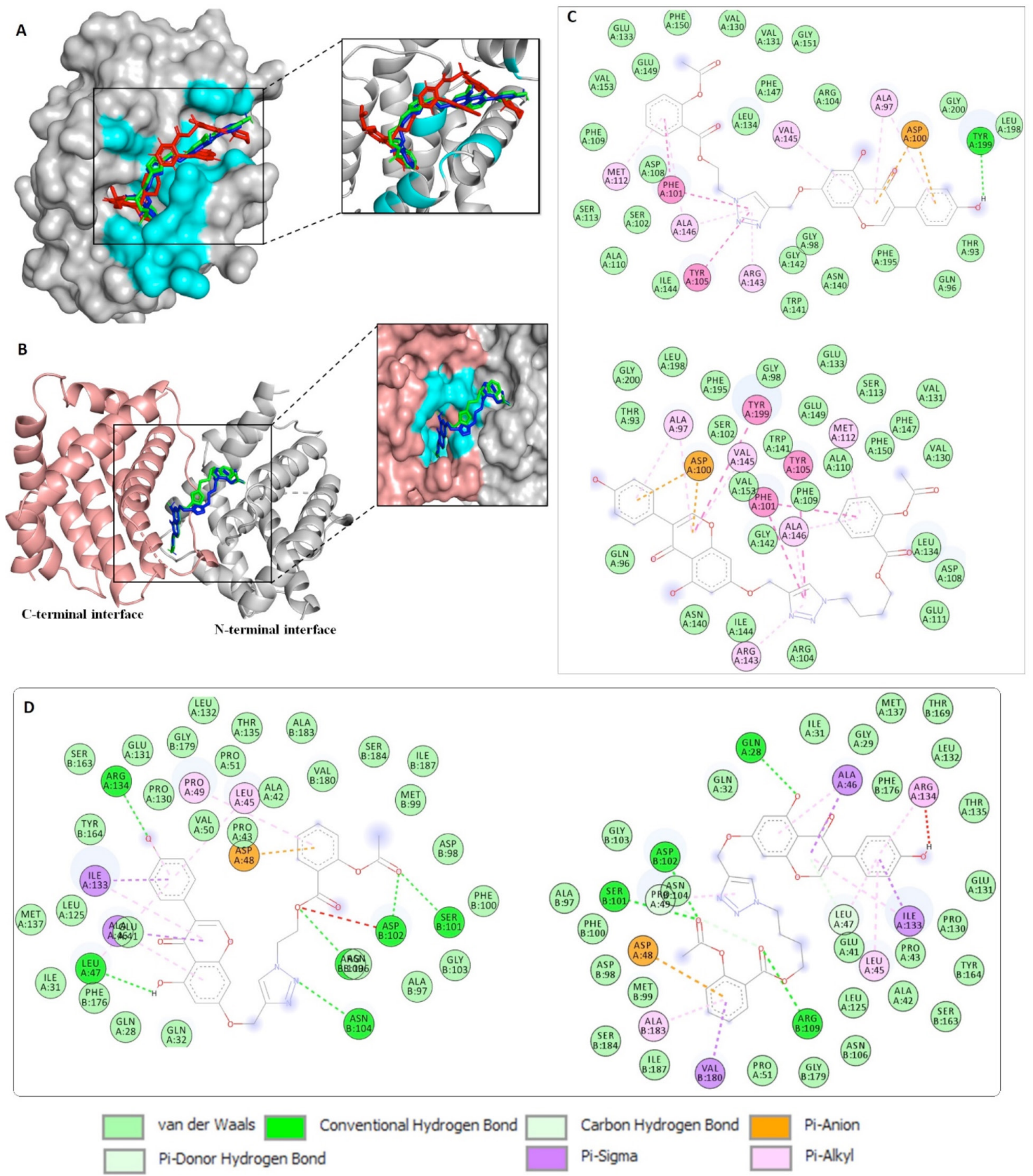

Figure 5. (A). Superposition of the best docked conformations of hybrids $4 \mathbf{4 a}$ (blue) and $\mathbf{4 c}$ (green) within the Bcl-2 active site. (B). Superposition of the best docked conformations of hybrids $\mathbf{4 a}$ (blue) and $\mathbf{4 c}$ (green) within the Bax dimer interface. (C). 2D protein-ligand plot interaction for $\mathbf{4 a}$ and $\mathbf{4 c}$ into the Bcl-2 active site. (D). 2D ligand-receptor interaction diagram for $4 \mathbf{a}$ and $\mathbf{4 c}$ within the Bax dimer interface. Catalytic residues are colored in cyan.

These computational findings suggest that inhibition of function human Polo-like kinase 1 (Plk1) could become a primary mode of action for active hybrids $\mathbf{4 a}$ (blue) and $\mathbf{4 c}$ (green). This presumably could explain the cytotoxic effect of these molecules on human adenocarcinoma cells (SW480).

\section{Hybrids $\mathbf{4 a}$ and $\mathbf{4 c}$ and the interaction with the Bcl-2/Bax system and $\mathrm{COX}-2$ receptor}

In addition to binding human Polo-like kinase 1 (Plk1), docking studies would suggest that hybrids $\mathbf{4 a}$ and $\mathbf{4 c}$ contribute to inhibit SW480 cell growth by modulating a second signaling pathway critically involved in colorectal cancer (CRC) development. As depicted in Table 2, hybrids $\mathbf{4 a}$ and $\mathbf{4 c}$ displayed a high-affinity binding for the human Bcl-2/Bax system in about $-10 \mathrm{kcal} / \mathrm{mol}$. Proapoptotic BAX and antiapoptotic Bcl-2 proteins are key regulators of apoptosis, which play an important role in resistance and expansion of neoplastic cells, particularly in human colorectal carcinomas (Nehls et al., 2007). Therapeutic strategies targeting the human Bcl-2/Bax system has emerged as an attractive alternative in patients with colorectal carcinomas and they are considered as a rational starting point to develop molecules that selectively trigger apoptosis of cancer cells. Figure 5A-D shows 
A
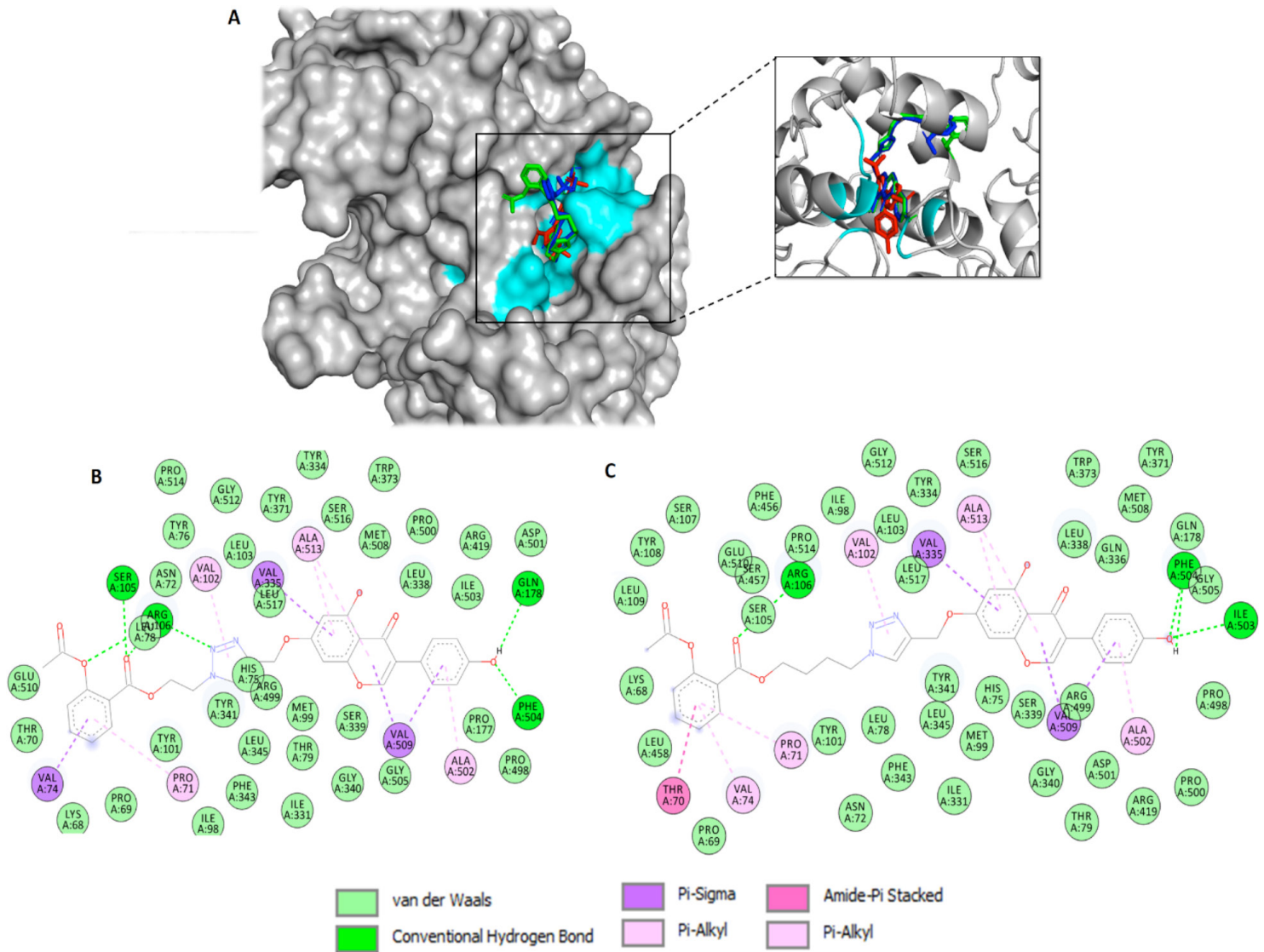

Figure 6. (A). Superposition of the best docked conformations of hybrids $4 a$ (blue) and $\mathbf{4 c}$ (green), and X-ray crystallographic pose of celecoxib (red) within the COX-2 active site. (B). 2D protein-ligand plot interaction for 4a into the COX-2 active site. (C). 2D ligandreceptor interaction diagram for $\mathbf{4} \mathbf{c}$ within the $\mathrm{COX}-2$ active domain. Catalytic residues are colored in cyan.

the most stable binding pose of hybrids $\mathbf{4 a}$ and $\mathbf{4} \mathbf{c}$ within the Bcl-2 catalytic domain ( -10.1 and $-9.9 \mathrm{kcal} / \mathrm{mol}$, respectively) as well as inside the Bax active site ( -9.8 and $-9.3 \mathrm{kcal} / \mathrm{mol}$, respectively). As illustrated in Figures $2 \mathrm{~A}-\mathrm{D}$, docking studies revealed that $\mathbf{4 a}$ and $\mathbf{4 c}$ have a strong interaction with residues of the $\mathrm{Bcl}-2 / \mathrm{Bax}$ system, fitting well into the binding pocket of the targeted receptors. For Bcl-2 protein, several amino acid residues have demonstrated to be essential for its function, among them, the role of Asp100, Phe101, Tyr105, Arg107, Asp108, Glu111, Met112, Arg143, Val145, Ala146, and Tyr199 in Bcl-2 active site has already been studied to develop more selective and effective molecules able to modulate Bcl-2 (Souers et al., 2013). On the other hand, human BAX protein exists in an inactive and dimeric conformation which is disrupted to release BAX monomers that are primed for a potent activation and apoptosis induction. The design of compounds able to disrupt the interactions of these swapped dimer structures could promote apoptosis in malignant cells (Garner et al., 2016). Activation site, which can be used to dissociate the BAX dimers, comprises ten key amino acid residues: Leu25, Leu26, Gln28, Gln 32, Ala34, Asp48, Asp102, Glu131, Ile133, and Arg134.

According to outlined above, this work found that hybrids 4a and $\mathbf{4 c}$ exhibit numerous intermolecular interactions with those essential residues for function of human $\mathrm{Bcl}-2 / \mathrm{Bax}$ system (Fig. 5C and D). Based on docking findings, titled compounds 4a and $4 \mathbf{c}$ bind to Bcl-2 receptor through H-bonds with key Tyr199, $\pi$-contacts with Phe101, Tyr105, Tyr199, $\pi$-anion interaction with charged residue Asp100, establishing several hydrophobic interactions which play an important role in stabilizing the binding event. Here, docking studies suggest that promising hybrids 4a and $\mathbf{4 c}$ could trigger apoptotic cell death in SW480 cells not only through inhibition of human Polo-like kinase 1 (Plk1), but also by modulation of the Bcl-2/Bax system.

Emerging evidence have proved that $\mathrm{COX}-2$ protein actively participates in proliferation, invasion, and adhesion of malignant cells, particularly in colorectal cancer. Therefore, novel candidates targeting COX-2 could suppresses tumor cell growth and invasion/migration and may be useful as an auxiliary therapeutic opportunity in colorectal cancer (CRC) patients (Huang et al., 2017; Ferrández et al., 2003; Sheng et al., 2020; Sinicrope et al., 2004). The COX-2 binding surface has been wellestablished. Thirteen residues form a large and deep pocket/cavity on the binding site of COX-2: Gln178, Val335, Leu338, Ser339, Gly340, Tyr341, Phe367, Ala502, Ile503, Phe504, Val509, Ala513, and Leu517 (Wang et al., 2010). Interestingly, when 4a and $\mathbf{4 c}$ were docked into the active site of COX-2 (PDB: 3LN1), showed a high binding affinity in about $-10 \mathrm{kcal} / \mathrm{mol}$ which was comparable to that anti-inflammatory drug celecoxib $(-9.6 \mathrm{kcal} /$ mol). The sampling provided by AutoDock showed that 4a and $4 \mathbf{c}$ were well-accommodated in the active site of $\mathrm{COX}-2$, as can be seen from Figure 6A. In fact, the best docking conformations obtained for hybrids $\mathbf{4 a}$ and $\mathbf{4 c}$ were in good agreement to that $\mathrm{X}$-ray pose of celecoxib. Furthermore, an exhaustive analysis 
of interaction fingerprint performance in DS Visualizer (Fig. 6B and $\mathrm{C}$ ) revealed that the plausible binding modes of compounds 4a and 4c form key hydrogen bond interactions with Gln178, Ile503, Phe504 playing an important role in the COX-2 function. Moreover, the molecular docking of $\mathbf{4 a}$ and $\mathbf{4} \mathbf{c}$ in the COX-2 shows interactions by $\pi$-contacts with the residuals comprising the catalytic region of the enzyme, such as Val335 and Val509. Finally, $\mathbf{4 a}$ and $\mathbf{4} \mathbf{c}$ formed numerous hydrophobic interactions at the active site of COX-2, which likely would play a crucial role in complex stabilizing upon ligand binding. Based on these findings, authors hypothesized that a probable modulation of COX-2 due to the action of ASA/Genistein hybrids $\mathbf{4 a}$ and $\mathbf{4 c}$ would be an important contributing factor to enhance the cytotoxic effect associated to these compounds against SW480 cells.

Taken altogether, docking results suggest that active hybrids $4 \mathbf{a}$ and $\mathbf{4 c}$ could trigger apoptotic SW480 cell death by attenuating the activity of the Polo-like kinase 1 (Plk1), which has a critical role in human colorectal carcinoma growing, thereby offering key insights into the cytotoxic mechanism of novel $\mathbf{4 a}$ and $\mathbf{4 c}$. Moreover, authors believe that the active hybrids $\mathbf{4 a}$ and 4c could be also acting via multitarget recognition of the proapoptotic Bcl-2/Bax system and COX-2, thereby could explain their cytotoxic effect on SW480 colorectal cancer cells. In this regard, hybrids $\mathbf{4 a}$ and $\mathbf{4 c}$ could represent a new class of multipotent therapeutic candidates for colorectal cancer treatment. However, these results clearly suggest that additional combined experimental and computational studies should be conducted to validate our docking statements.

\section{Pharmacokinetic studies and in silico ADME/Tox modeling}

\section{Lipinski's rule and drug-likeness evaluation}

Early theorical calculations of drug-likeness parameters have the most profound influence during novel anti-cancer hits development, concerning to their pharmacokinetic quality. Accordingly, pharmacokinetic properties based on the structure of promising oncology compounds could help to find more effective and safer candidates for further preclinical testing. To assess the druglikeness of the most active synthesized compounds $\mathbf{4 a}$ and $\mathbf{4} \mathbf{c}$, in silico pharmacokinetics studies were conducted by using free web tools for cheminformatics analysis, and results are summarized in Table 3. Thus, ten biopharmaceutical and pharmacokinetic descriptors such as MW, H-bond donor/acceptor, number of rotatable bonds, TPSA, octanol/water partition coefficient $\left(\log \mathrm{P}_{\mathrm{o} / \mathrm{w}}\right)$, gastrointestinal absorption (GI), permeability values across traditional Caco-2 and MDCK cells models and the binding-serum albumin (as $\operatorname{LogK}_{\mathrm{HSA}}$ ) were estimated for the titled hybrids, and data are shown in Table 3.

Interestingly, favorable pharmacokinetics indices were founded for the hybrids compared to $95 \%$ of US-marketed drugs. According to Lipinski's guidelines (1997), the top-two hybrids 4a and $\mathbf{4 c}$ displayed an optimal physicochemical profile (no more than one violation is acceptable), which suggests these compounds are likely to be orally active anti-cancer candidates. Despite those high degrees of lipophilicity (expressed as $\log \mathrm{P}_{\mathrm{o} / \mathrm{w}}$ ) that were estimated for hybrids 4a and 4c (3.025 and 5.597, respectively), both fit well within the optimal range for lipid-based formulations (-2.0 to 6.0) (Ditzinger et al., 2019).
Table 3. Lipinski's rule and pharmacokinetic indices for the active hybrids $\mathbf{4 a}$ and $\mathbf{4 c}$.

\begin{tabular}{|c|c|c|}
\hline Properties & $4 a$ & $4 c$ \\
\hline $\mathrm{MW}^{\mathrm{a}}$ & 557.515 & 585.569 \\
\hline TPSA $^{\mathrm{b}}$ & 175.154 & 180.608 \\
\hline n-Rot Bond & 11 & 13 \\
\hline $\mathrm{n}-\mathrm{ON}^{\mathrm{c}}$ & 10 & 10 \\
\hline $\mathrm{n}-\mathrm{OHNH}{ }^{\mathrm{d}}$ & 2 & 2 \\
\hline $\log \mathrm{P}_{\mathrm{o} / \mathrm{w}}{ }^{\mathrm{e}}$ & 3.809 & 4.244 \\
\hline $\log \mathrm{K}_{\mathrm{HSA}}{ }^{\mathrm{f}}$ & 0.305 & 0.504 \\
\hline Caco-2 $(\mathrm{nm} / \mathrm{s})^{\mathrm{g}}$ & 66 & 35 \\
\hline App. $M D C K(\mathrm{~nm} / \mathrm{s})^{\mathrm{h}}$ & 26 & 13 \\
\hline$\% G I^{\mathrm{i}}$ & 56 & 54 \\
\hline Lipinski's violations $(\leq 1)$ & 1 & 1 \\
\hline PAINS $^{j}$ & 0 & 0 \\
\hline
\end{tabular}

${ }^{a}$ Molecular weight of the compound (150-500).

bolar surface area $\left(P S A, \AA^{2}\right)(7.0-200)$.

${ }^{c} n-O N$ number of hydrogen bond acceptors $<10$.

${ }^{\mathrm{d}} \mathrm{n}-\mathrm{OHNH}$ number of hydrogens bonds donors $\leq 5$.

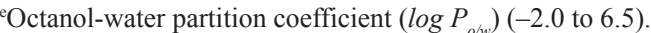

${ }^{\mathrm{f}}$ Binding-serum albumin $\left(\log K_{H S A}\right)(-1.5$ to 1.2$)$.

${ }^{8}$ Human intestinal permeation $(<25$ poor, $>500$ great $)$.

${ }^{\mathrm{h}}$ Madin-Darby canine kidney $(M D C K)$ cells permeation $(<25$ poor, $>500$ great). ${ }^{\mathrm{i}} \%$ Human oral gastrointestinal $(G I)$ absorption ( $<25 \%$ is poor).

IIdentification of potentially problematic fragments for pan-assay interference compounds (PAINS).

In addition, active hybrids $\mathbf{4 a}$ and $\mathbf{4 c}$ exhibited optimal human intestinal absorption (\%GI) numbers greater than $50 \%$, this means that upon oral administration, the target hybrids could have a greater chance to be absorbed throughout the gastrointestinal segments. This parameter was also checked through calculations by using transmembrane permeation across traditional Caco-2 and MDCK cells lines. Currently, these cells lines are the most widely used in vitro model at investigating of permeability of drugs through intestinal barrier in the development of oral pharmaceutical products (Broccatelli et al., 2016; Pham-The et al., 2018; Press et al., 2008). Thus, hybrids 4a and 4c showed to have good permeability with values in the range of 13 to 66 $\mathrm{nm} / \mathrm{s}$, demonstrating that these compounds could be moderately absorbed into the gastrointestinal tract.

For hybrids $\mathbf{4 a}$ and $\mathbf{4 c}$, another key biopharmaceutical parameter, named Polar Surface Area (TPSA), which is a good predictor of drug-membrane interactions and permeation across biological membranes was also investigated. Thus, TPSA measurements for hybrids $\mathbf{4 a}$ and $\mathbf{4 c}$ showed acceptable therapeutic values of 175.154 and $180.608 \AA^{2}$, respectively, which in turn suggests that these compounds would penetrate tumor cells more efficiently.

In addition, the ability of the hybrids to bind to human serum albumin (calculated as $\log \mathrm{K}_{\mathrm{HSA}}$ ) was investigated, which is the most significant parameter for distribution and transport of drug and drug-like molecules in the systemic circulation. For therapeutic uses, $\log \mathrm{K}_{\mathrm{HSA}}$ values in the range of -1.5 to 1.5 are recommended for potential drugs (Colmenarejo et al., 2003; Zhivkova et al., 2015). It was then estimated that compounds $\mathbf{4 a}$ and $\mathbf{4 c}$ display affinities with 
HSA within the recommended therapeutic values, finding $\log \mathrm{K}_{\mathrm{HSA}}$ numbers between 0.305 and 0.504 , respectively. Finally, the panassay interference compounds (PAINS) filter which provide early alerts for potential toxicity as part of successful drug discovery, showed that novel hybrids can be regarded as valid development starting points in cancer drug discovery.

Taken altogether, merging ASA and Genistein subunits into a unique structural core provide active compounds with optimal pharmacokinetic profile, making this scaffold promissory to develop novel drug-candidates against colorectal cancer.

\section{OSIRIS drug-likeness score and toxicity calculations}

Prediction of drug-relevant properties and overall toxicity of bioactive ASA-Genistein hybrids $\mathbf{4 a}$ and $\mathbf{4} \mathbf{c}$ were carried out by using the OSIRIS property explorer (a free webbased program), which is a powerful web server to predict physicochemical and toxicological molecular properties (Table 4). Calculations were used to estimate the risks of side effects, such as mutagenicity, tumorigenic risk, irritant, and reproductive effects, as well as drug-like index to quantify the drug-like "degree." The toxicity risk predictions locate substructures within a molecule that could enhance the risks of adverse drug reactions on a cell, organ, or organisms. Notably, the toxicity risk assessment suggested that hybrids $\mathbf{4 a}$ and $\mathbf{4 c}$ would display low or null toxicity regarding mutagenicity, tumorigenicity, irritant effect, but unfortunately some molecular features in the chemical structure of the synthesized compounds were identified as irritating on reproductive system.

On the other hand, drug-likeness model score used by the OSIRIS web tool suggests the potential therapeutic properties for top-hybrids $\mathbf{4 a}$ and $\mathbf{4 c}$ in comparison with marketed drugs. The OSIRIS database contains 5300 substructure fragments, with their associated drug-likeness scores, which have been identified in 15000 commercially available chemical compounds and 3300 US-traded drugs. For every fragment, after correction and removal of highly redundant fragments, a drug-likeness score is assigned based on the frequency of occurrence of the fragment. The diagram of the distribution of drug-likeness values for the marketed drugs versus the commercially available chemicals shows that about $80 \%$ of traded drugs have positive values of the drug-likeness score while the commercial compounds (non-drug related compounds) have negative values. Hence, a positive number for the drug-likeness score indicates that the compound is made up mostly of building blocks (fragments) that are commonly found in marketed drugs. As depicted in Table 4, hybrids compounds 4a and $\mathbf{4 c}$ display positive drug-likeness values of 0.77 and 0.67 , respectively. These findings suggest that these compounds could be safe drug candidates, or that their structures could be used as models for scaffold-based drug discovery in colorectal cancer chemotherapy.

Data obtained by the OSIRIS web tool suggest favorable outcomes of safety and drug-likeness for the most bioactive ASA-Genistein hybrids $\mathbf{4 a}$ and $\mathbf{4 c}$, which in turn means that these compounds have great chance to advance through the earlier preclinical testing steps as well as serve as attractive candidates for future drug development treatments focused on the initiation, progression, and migration linked with colorectal cancer.
Table 4. Drug-likeness score and toxicity risk calculations of hybrids $4 \mathbf{a}$ and $\mathbf{4} \mathbf{c}$ based on OSIRIS property explorer.

\begin{tabular}{|c|c|c|}
\hline Toxicity risk ${ }^{a}$ & $4 a$ & $4 c$ \\
\hline Mutagenicity & Green & Green \\
\hline Tumorigenic & Green & Green \\
\hline Irritant effect & Green & Green \\
\hline Reproductive effect & Red & Red \\
\hline Drug-likeness ${ }^{\mathrm{b}}$ & 0.77 & 0.67 \\
\hline
\end{tabular}

${ }^{\mathrm{a}} \mathrm{Green}$ (no risk or low risk), yellow (medium risk), and red (higher toxicity risk).

${ }^{\mathrm{b}} \mathrm{A}$ positive number indicates a promising active compound.

\section{In vitro assessment of metabolic stability}

From the therapeutic view, metabolism studies of a potential drug-candidate represent one of the most important pharmacokinetic and biochemical challenges to consider during the early stages of drug discovery (Zhang et al., 2018). In this context, metabolic stability is a key pharmacokinetic parameter which often provides a valuable information about bioavailability, half-life, intrinsic clearance, and volume of distribution of a potential clinical drug candidate (Słoczyńska et al., 2019). Thus, metabolic stability data of a drug-like molecule may offer key evidence about its susceptibility to be bio-transformed. However, in vitro and in vivo metabolic stability studies often requires laborious manual manipulations, automated, and robust assays that tend to increase the cost- and time-effectiveness of the product development cycle. In contrast, chemoinformatics tools provide a low-cost, high-throughput, and accurate approach for a rapid screening of metabolic stability of large compound library identify as potential drug-candidates. MetStabOn is a powerful web server for the qualitative prediction of metabolic stability based on the application of machine learning methods and separate models for human, rat, and mouse. MetStabOn not only expresses the metabolic stability of a hit-compound as half-lifetime and clearance, but values are assigned within three metabolic stability classes [expressed as $\mathrm{T}_{1 / 2}$, low ( $\leq 0.6$ hours), medium (0.6-2.32 hours), or high ( $>2.32$ hours)], which are based on in vitro assays performed on liver microsomes referring to human, rat, and mouse experiments (Podlewska et al., 2018). Herein, MetStabOn was used to estimate the metabolic stability for top-two hybrids $\mathbf{4 a}$ and $\mathbf{4 c}$, aiming to provide early insight into the first-pass metabolism and bioavailability. The predicted metabolic stabilities values for hybrids $\mathbf{4 a}$ and $\mathbf{4 c}$ are summarized in Table 5.

Interestingly, these compounds exhibited favorable numerical values of metabolic stability in human, rat, and mouse models with half-lifetimes values belonging to medium (0.6-2.32 hours) and high ( $>2.32$ hours) stability class. More importantly, referring to human model, in which human liver microsomes were used, the obtained $T_{1 / 2}$ stability values for ASA-Genistein hybrids 4a and $\mathbf{4 c}$ varied from 1.001 to 1.033 hours, indicating that these compounds would have a medium metabolic stability. Similarly, the predictions of metabolic stability with mouse model produced on $T_{1 / 2}$ liver microsomes data revealed that hybrids $4 \mathbf{a}$ and $\mathbf{4} \mathbf{c}$ may have median values of half-lifetimes. In view of the above findings regarding to metabolic stability, novel ASA-Genistein hybrids 4a 
Table 5. Predicted metabolic stabilities for hybrids $4 \mathbf{a}$ and $4 \mathbf{c}$

\begin{tabular}{cccc}
\hline Hybrid & \multicolumn{3}{c}{$\boldsymbol{T}_{1 / 2}(\text { hour })^{\mathrm{a}, \mathrm{b}}$} \\
\cline { 2 - 4 } & Human & Rat & Mouse \\
\hline 4a & 1.001 & 0.461 & 1.150 \\
$\mathbf{4 c}$ & 1.033 & 4.526 & 1.441 \\
\hline
\end{tabular}

${ }^{\mathrm{a} E s t i m a t e d ~ m e t a b o l i c ~ s t a b i l i t y ~ p r o d u c e d ~ o n ~ l i v e r ~ m i c r o s o m e s . ~}$

${ }^{\mathrm{b}}$ Metabolic stability cutoffs as follows ( $T_{1 / 2}$ expressed in hours): $\leq 0.6$ (Low), $0.6-2.32$ (Medium), and $>2.32$ (High).

and $\mathbf{4 c}$ apparently would have low hepatic first-pass metabolism and sufficient time to trigger the desired therapeutic response.

\section{CONCLUSION}

In this work, different hybrids based on ASA and genistein were synthesized for the first time. Besides, the chemopreventive potential of these molecules using a $2 \mathrm{D}$ in vitro model of colorectal cancer was evaluated. Our findings suggest that hybrids based on ASA and genistein could involve selective growth inhibition, suggesting an increase toward cell death or a cell cycle arrest, leading to changes in size and shape after treatment, with better and selective results than parental compounds and the conventional chemotherapeutic (5-FU), highlighting the potential of the strategy based on molecular hybridization. Additionally, docking studies show markedly binding affinities when active $\mathbf{4 a}$ and $\mathbf{4 c}$ were docked against Polo-like kinase 1 protein in about $-10 \mathrm{kcal} / \mathrm{mol}$, as well as against Bcl-2/Bax system and COX-2 (in the range of -10.1 to $-9.3 \mathrm{kcal} / \mathrm{mol}$ ), suggesting that these compounds apparently may induce synergistic cytotoxic effect in SW480 cells due to multitarget interactions with these proteins. Finally, in silico drug-likeness evaluation was accessed for active hybrids $\mathbf{4 a}$ and $\mathbf{4 c}$, and results suggest that these compounds have acceptable pharmacokinetics indices to be considered drug-like scaffolds in medicinal chemistry. All these biological and molecular modeling data obtained from this study suggest that ASA/Genistein hybrids (4a and $\mathbf{4 c}$ ) could be valuable therapeutic scaffolds for further studies to identify new alternatives for the treatment of colorectal cancer.

\section{ACKNOWLEDGMENT}

This research was funded by the University of Antioquia, MINCIENCIAS, MINEDUCATION, MINCIT and ICETEX, through the Program Ecosistema Científico Cod. FP44842-2112018 project number 58537 . The authors wish to thank Dr. Sabina Podlewska for their work in estimated metabolic stability (Institute of Pharmacology, Polish Academy of Sciences, Department of Medicinal Chemistry).

\section{AUTHOR CONTRIBUTIONS}

L.G.: synthesis and characterization of hybrid molecules. G.M.: evaluation of biological activities and formal analysis. A.H.-R.: conceptualization, methodology, validation, evaluation of biological activities and formal Analysis, writing-original Draft, writing-review \& editing. A.F-Y: in silico study and writing-original Draft. W.C.-G.: conceptualization, methodology, resources, writingoriginal draft, writing-review \& editing, supervision, project administration, funding acquisition. All authors have read and agreed to the published version of the manuscript.

\section{FUNDING}

This research was funded by the University of Antioquia, MINCIENCIAS, MINEDUCATION, MINCIT and ICETEX, through the Program Ecosistema Científico Cod. FP44842-2112018 project number 58537.

\section{CONFLICTS OF INTEREST}

The authors declare no conflicts of interest.

\section{ETHICAL APPROVAL}

This study does not involve the use of animals or human subjects.

\section{REFERENCES}

Alqahtani Z, Jamali F. Clinical outcomes of aspirin interaction with other non-steroidal anti-inflammatory drugs: a systematic review. J Pharm Pharm Sci, 2018; 21(1s):29854; doi: 10.18433/jpps29854.

Avivi D, Moshkowitz M, Detering E, Arber N. The role of lowdose aspirin in the prevention of colorectal cancer. Expert Opin Ther Targets 2012; 16(Suppl 1):S51-62; doi:10.1517/14728222.2011.647810.

Broccatelli F, Salphati L, Plise E, Cheong J, Gobbi A, Lee ML, Aliagas I. Predicting passive permeability of drug-like molecules from chemical structure: where are we? Mol Pharm, 2016; 13(12):4199-208; doi:10.1021/acs.molpharmaceut.6b00836

Cardona-G W, Herrera-R A, Castrillón-L W, Ramírez-Malule $\mathrm{H}$. Chemistry and anticancer activity of hybrid molecules and derivatives based on 5-fluorouracil. Curr Med Chem, 2021; 28:1-53; doi: 10.2174/092 9867328666210211164314

Cardona-G W, Yepes AF, Herrera-R A. Hybrid Molecules: promising compounds for the development of new treatments against Leishmaniasis and Chagas disease. Curr Med Chem, 2018; 25:3615-57; do i:10.2174/0929867325666180309111428.

Chae HS, Xu R, Won JY, Chin YW, Yim H. Molecular targets of genistein and its related flavonoids to exert anticancer effects. Int J Mol Sci, 2019; 20(10):2420-9; doi:10.3390/ijms20102420.

Colmenarejo G. In silico prediction of drug-binding strengths to human serum albumin. Med Res Rev, 2003; 23(3):275-301; doi:10.2174/1 381612821666150302113710

Daina A, Michielin O, Zoete V. SwissADME: A free web tool to evaluate pharmacokinetics, drug-likeness and medicinal chemistry friendliness of small molecules. Sci Rep, 2017; 7:1-13; doi:10.1038/ srep42717.

Ding Y, Li S, Ge W, Liu Z, Zhang X, Wang M, Chen T, Chen Y, Zhang Q. Design and synthesis of parthenolide and 5-fluorouracil conjugates as potential anticancer agents against drug resistant hepatocellular carcinoma. Eur J Med Chem, 2019; 183:111706; doi:10.1016/j.ejmech.2019.111706

de Oliveira Pedrosa M, Duarte da Cruz RM, de Oliveira Viana J, de Moura RO, Ishiki HM, Barbosa Filho JM, Diniz MF, Scotti MT, Scotti L, Bezerra Mendonca FJ. Hybrid compounds as direct multitarget ligands: a review. Curr Top Med Chem, 2017; 17:1044-79; doi:10.2174/15680266 16666160927160620.

Ditzinger F, Price DJ, Ilie AR, Köhl NJ, Jankovic S, Tsakiridou G, Aleandri S, Kalantzi L, Holm R, Nair A, Saal C, Griffin B, Kuentz M. Lipophilicity and hydrophobicity considerations in bio-enabling oral formulations approaches - a PEARRL review. J Pharm Pharmacol, 2019; 71:464-82; doi:10.1111/jphp.12984.

Ferrández A, Prescott S, Burt RW. COX-2 and colorectal cancer. Curr Pharm Des, 2003; 9(27):2229-51; doi:10.2174/1381612033454036

Ganai AA, Farooqi H. Bioactivity of genistein: a review of in vitro and in vivo studies. Biomed Pharmacother, 2015; 76:30-8; doi:10.1016/j.biopha.2015.10.026

Garner TP, Reyna DE, Priyadarshi A, Chen HC, Li S, Wu Y, Ganesan YT, Malashkevich VN, Cheng EH, Gavathiotis E. An autoinhibited 
dimeric form of BAX regulates the BAX activation pathway. Mol Cell, 2016; 63(3):485-97; doi:10.1016/j.molcel.2016.06.010

Global Cancer Statistics 2020: GLOBOCAN Estimates of Incidence and Mortality Worldwide for 36 Cancers in 185 Countries. CA Cancer J Clin, 2021; 0:1-41. Available via https://acsjournals.onlinelibrary. wiley.com/doi/10.3322/caac.21660 (Accessed June 2021).

Hashemi Goradel N, Najafi M, Salehi E, Farhood B, Mortezaee K. Cyclooxygenase-2 in cancer: a review. J Cell Physiol, 2019; 234(5):568399; doi:10.1002/jcp. 27411

Herrera-RA, Castrillón W, Otero E, Ruiz E, Carda M, Agut R, Naranjo T, Moreno G, Maldonado ME, Cardona-G W. Synthesis and antiproliferative activity of 3- and 7-styrylcoumarins. Med Chem Res, 2018; 27:1893-905.

Huang WW, Hsieh KP, Huang RY, Yang YH. Role of cyclooxygenase- 2 inhibitors in the survival outcome of colorectal cancer patients: a population-based cohort study. Kaohsiung J Med Sci, 2017; 33(6):308-14; doi:10.1016/j.kjms.2017.03.004

Jiang W, Yan Y, Chen M, Luo G, Hao J, Pan J, Hu S, Guo P, Li W, Wang R, Zuo Y, Sun Y, Sui S, Yu W, Pan Z, Zou K, Zheng Z, Deng W, Wu $\mathrm{X}$, Guo W. Aspirin enhances the sensitivity of colon cancer cells to cisplatin by abrogating the binding of NF- $\mathrm{kB}$ to the COX-2 promoter. Aging (Albany NY), 2020; 12(1):611-27; doi: 10.18632/aging.102644

Kothe M, Kohls D, Low S, Coli R, Cheng AC, Jacques SL, Johnson TL, Lewis C, Loh C, Nonomiya J, Sheils AL, Verdries KA, Wynn TA, Kuhn C, Ding YH. Structure of the catalytic domain of human pololike kinase 1. Biochemistry, 2007; 46(20):5960-71; doi:10.1021/bi602474j Lai MY, Huang JA, Liang ZH, Jiang HX, Tang GD. Mechanisms underlying aspirin-mediated growth inhibition and apoptosis induction of cyclooxygenase- 2 negative colon cancer cell line SW480. World J Gastroenterol 2008;14(26):4227-4233; doi: 10.3748/wjg.14.4227

LeCher JC, Diep N, Krug PW, Hilliard JK. Genistein has antiviral activity against Herpes B virus and acts synergistically with antiviral treatments to reduce effective dose. Viruses, 2019; 11(6):499; doi:10.3390/v11060499

Lin LP, Wu FH, Liang JY. The first examples of ilexgenin A hybrids as a new class of multi-potent, anti-platelet agents. Chin Chem Lett, $2013 ; 24: 723-6$.

Lipinski CA, Lombardo F, Dominy BW, Feeney PJ. Experimental and computational approaches to estimate solubility and permeability in drug discovery and development settings. Adv Drug Deliv Rev, 1997; 23:3-25; doi:10.1016/s0169-409x(00)00129-0

Luo Y, Wang SX, Zhou ZQ, Wang Z, Zhang YG, Zhang Y, Zhao P. Apoptotic effect of genistein on human colon cancer cells via inhibiting the nuclear factor-kappa B (NF-kB) pathway. Tumour Biol, 2014; 35(11):11483-8; doi:10.1007/s13277-014-2487-7

Mastuda H, Morikawa T, Ueda K, Managi H, Yoshikawa M. Structural requirements of flavonoids for inhibition of antigen-Induced degranulation, TNF- $\alpha$ and IL-4 production from RBL-2H3 cells. Bioorg Med Chem, 2002; 10:3123-8; doi:10.1016/S0968-0896(02)00227-4

McQuade RM, Bornstein JC, Nurgali K. Anti-colorectal cancer chemotherapy-induced diarrhoea: current treatments and side effects. Int J Clin Med, 2014; 5:393-406; doi:10.4236/ijcm.2014.57054

Morris GM, Goodshell DS, Halliday RS, Huey R, Hart WE, Belew RK, Olson AJ. Docking using a Lamarckian genetic algorithm and empirical binding free energy function. J Comput Chem, 1998; 19:1639-62; doi:10.1002/ (SICI)1096-987X(19981115)19:14<1639::AID-JCC10>3.0.CO;2-B

Morris GM, Huey R, Lindstrom W, Sanner MF, Belew RK, Goodsell DS, Olson AJ. AutoDock4 and AutoDockTools4: Automated docking with selective receptor flexibility. J Comput Chem, 2009; 30(16):2785-91; doi: $10.1002 / j c c .21256$

Nehls O, Okech T, Hsieh CJ, Enzinger T, Sarbia M, Borchard F, Gruenagel HH, Gaco V, Hass HG, Arkenau HT, Hartmann JT, Porschen R, Gregor M, Klump B. Studies on p53, BAX and Bcl-2 protein expression and microsatellite instability in stage III (UICC) colon cancer treated by adjuvant chemotherapy: major prognostic impact of proapoptotic BAX. $\mathrm{Br}$ J Cancer, 2007; 96(9):1409-18; doi:10.1038/sj.bjc.6603728.
Rahman Mazumder MA, Hongsprabhas P. Genistein as antioxidant and antibrowning agents in in vivo and in vitro: a review. Biomed Pharmacother, 2016; 82:379-92; doi: 10.1016/j.biopha.2016.05.023

Rejhova A, Opattova A, Cumov A, Slíva D, Vodicka P. Natural compounds and combination therapy in colorectal cancer treatment. Eur J Med Chem, 2018; 144:582-94; doi:10.1016/j.ejmech.2017.12.039

Satish A, Farha Syeda S, Urooj A. Quantification of flavonoids by UPLC-MS and its antibacterial activity from Brassica oleracea var. Capitata L. GSC Biol Pharm Sci, 2018; 05(01):109-14; doi:10.30574/gscbps.2018.5.1.0105

Satya P, Indira J, Gayathri C, Vijayalekshmi N, Syng-Ook L, Stephen S. Aspirin inhibits colon cancer cell and tumor growth and downregulates specificity protein (Sp) transcription factors. PLoS One, 2012; 7(10):e48208. Published online 2012 Oct 26; doi: 10.1371/journal. pone. 0048208

Sheng J, Sun H, Yu FB, Li B, Zhang Y, Zhu YT. The role of cyclooxygenase-2 in colorectal cancer. Int J Med Sci, 2020; 17(8):1095101; doi:10.7150/ijms.44439

Shin SB, Woo SU, Chin YW, Jang YJ, Yim H. Sensitivity of TP53-mutated cancer cells to the phytoestrogen genistein is associated with direct inhibition of Plk1 activity. J Cell Physiol, 2017; 232(10):2818-28; doi:10.1002/jcp. 25680

Shinde V, Mhaske PC, Singh A, Sarkar D, Mahulikar P Synthesis and biological evaluation of new 4-(4-(1-benzyl-1H-1,2,3triazol-4-yl)phenyl)-2-phenyl thiazole derivatives. J Heterocyclic Chem, 2019; 56:3093-101; doi:10.1002/jhet.3708.

Sinicrope FA, Gill S. Role of cyclooxygenase-2 in colorectal cancer. Cancer Metastasis Rev, 2004; 23(1-2):63-75; doi:10.1023/a:1025863029529.

Słoczyńska K, Gunia-Krzyżak A, Koczurkiewicz P, WójcikPszczoła K, Żelaszczyk D, Popiół J, Pękala E. Metabolic stability and its role in the discovery of new chemical entities. Acta Pharm, 2019; 69(3):345-61; doi:10.2478/acph-2019-0024.

Souers AJ, Leverson JD, Boghaert ER, Ackler SL, Catron ND, Chen J, Dayton BD, Ding H, Enschede SH, Fairbrother WJ, Huang DC, Hymowitz SG, Jin S, Khaw SL, Kovar PJ, Lam LT, Lee J, Maecker HL, Marsh KC, Mason KD, Mitten MJ, Nimmer PM, Oleksijew A, Park CH, Park CM, Phillips DC, Roberts AW, Sampath D, Seymour JF, Smith ML, Sullivan GM, Tahir SK, Tse C, Wendt MD, Xiao Y, Xue JC, Zhang H, Humerickhouse RA, Rosenberg SH, Elmore SW. ABT-199, a potent and selective BCL-2 inhibitor, achieves antitumor activity while sparing platelets. Nat Med, 2013; 19(2):202-8; doi:10.1038/nm.3048

Spagnuolo C, Moccia S, Russo GL. Anti-inflammatory effects of flavonoids in neurodegenerative disorders. Eur J Med Chem, 2018; 153:105-15; doi: 10.1016/j.ejmech.2017.09.001

Pérez JM, Maldonado ME, Rojano B, Alzate F, Sáez J, Cardona W. Comparative antioxidant, antiproliferative and apoptotic effects of Ilex laurina and Ilex paraguariensis on colon cancer cells. Trop J Pharm Res, 2014; 13:1279-86.

Pham-The H, Cabrera-Pérez MÁ, Nam NH, Castillo-Garit JA, Rasulev B, Le-Thi-Thu H, Casañola-Martin GM. In silico assessment of ADME properties: advances in Caco-2 cell monolayer permeability modeling. Curr Top Med Chem, 2018; 18(26):2209-29; doi:10.2174/1568 026619666181130140350

Podlewska S, Kafel R. MetStabOn-online platform for metabolic stability predictions. Int J Mol Sci, 2018; 19:1040-56; doi:10.3390/ ijms19041040

Pointet AL, Taieb J. Cáncer de colon. EMC — Tratado De Med, 2017; 21:1-7; doi:10.1016/S1636-5410(16)81792-4

Press B, Di Grandi D. Permeability for intestinal absorption: Caco-2 assay and related issues. Curr Drug Metab, 2008; 9(9):893-900; doi: $10.2174 / 138920008786485119$

Takahashi T, Sano B, Nagata T, Kato H, Sugiyama Y, Kunieda K, Kimura M, Okano Y, Saji S. Polo-like kinase 1 (PLK1) is overexpressed in primary colorectal cancers. Cancer Sci, 2003; 94(2):148-52; doi:10.1111/j.1349-7006.2003.tb01411.x 
Trott O, Olson AJ. AutoDock Vina: improving the speed and accuracy of docking with a new scoring function, efficient optimization, and multithreading. J Comput Chem, 2010; 31(2):455-61; doi:10.1002/ jec. 21334

Tuli HS, Tuorkey MJ, Thakral F, Sak K, Kumar M, Sharma AK, Sharma U, Jain A, Aggarwal V, Bishayee A. Molecular mechanisms of action of genistein in cancer: recent advances. Front Pharmacol, 2019; 10:1336-41; doi:10.3389/fphar.2019.01336

Wang XY, Clubbs EA, Bomser JA. Genistein modulates prostate epithelial cell proliferation via estrogen-and extracellular signalregulated kinase-dependent pathways. J Nutr Biochem, 2006; 17:204-10; doi:10.1016/j.jnutbio.2005.07.005.

Wang JL, Limburg D, Graneto MJ, Springer J, Hamper JR, Liao S, Pawlitz JL, Kurumbail RG, Maziasz T, Talley JJ, Kiefer JR, Carter J. The novel benzopyran class of selective cyclooxygenase-2 inhibitors. Part 2: the second clinical candidate having a shorter and favorable human half-life. Bioorg Med Chem Lett, 2010; 20(23):7159-63; doi:10.1016/j. bmcl.2010.07.054

Yang Y, Zang A, Jia Y, Shang Y, Zhang Z, Ge K, Zhang J, Fan W, Wang B. Genistein inhibits A549 human lung cancer cell proliferation via miR-27a and MET signaling. Oncol Lett, 2016; 12(3):2189-93; doi:10.3892/ol.2016.4817

Zhang Z, Chen FL, Shang L. Advances in antitumor effects of NSAIDs. Cancer Manag Res, 2018; 10:4631-40; doi:10.2147/CMAR. S175212

Zhang Zh, Tang W. Drug metabolism in drug discovery and development. Acta Pharm Sin B, 2018; 8(5):721-32; doi:10.1016/j. apsb.2018.04.003.

Zhivkova ZD. Studies on drug-human serum albumin binding: the current state of the matter. Curr Pharm Des, 2015; 21(14):1817-30; doi $: 10.2174 / 1381612821666150302113710$
Zhang J, Su H, Li Q, Li J, Zhao Q. Genistein decreases A549 cell viability via inhibition of the PI3K/AKT/HIF- $1 \alpha / \mathrm{VEGF}$ and NF- $\mathrm{\kappa B} / \mathrm{COX}-2$ signaling pathways. Mol Med Rep, 2017; 15(4):2296-302; doi:10.3892/ mmr.2017.6260

Zhou P, Wang C, Hu Z, Chen W, Qi W, Li A. Genistein induces apoptosis of colon cancer cells by reversal of epithelial-to-mesenchymal via a Notch1/NF-kB/slug/E-cadherin pathway. BMC Cancer, 2017; 17(1):81322; doi:10.1186/s12885-017-3829-9

How to cite this article: Gómez-R L, Moreno-Q G, Herrera-R A, Castrillón-L W, Yepes AF, Cardona-G W. New hybrid scaffolds based on ASA/genistein: Synthesis, cytotoxic effect, molecular docking, drug-likeness, and in silico ADME/Tox modeling. J Appl Pharm Sci, 2022; 12(02):015-030. 